\title{
Hydrogen-Deuterium Exchange in Basic Near-critical and Supercritical Media: An Experimental and Theoretical Study.
}

Pia R. Alburquerque ${ }^{\dagger}$, Ramu Ramachandran ${ }^{\ddagger}$, Thomas Junk ${ }^{\ddagger}$ and Tolga N. V. Karsili ${ }^{¥ *}$

${ }^{\dagger}$ Department of Chemistry, Grambling State University, Grambling, Louisiana 71245, U.S.A.

Institute for Micromanufacturing, Louisiana Tech University, Ruston, Louisiana 71272, U.S.A.

${ }^{¥}$ Department of Chemistry, University of Louisiana at Lafayette, Lafayette, Louisiana 70504,

$$
\text { U.S.A. }
$$

\section{Supporting Information}

\section{Table of Contents}

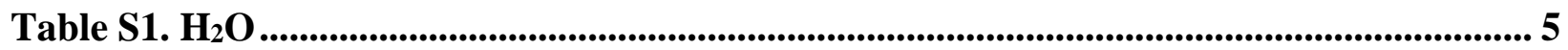

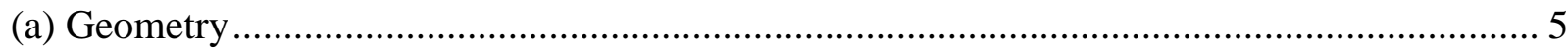

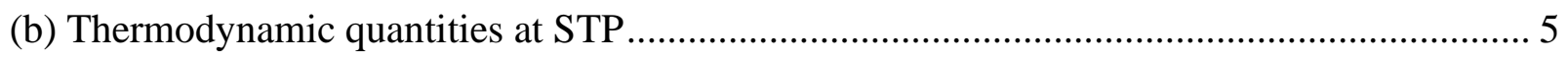

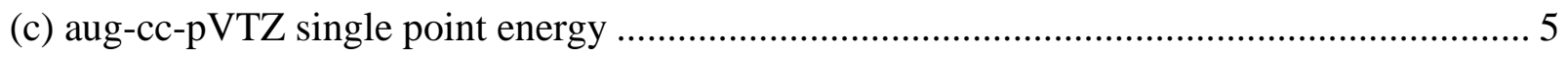

(d) Energy in the aqueous phase at STP (SMD model) ................................................. 5

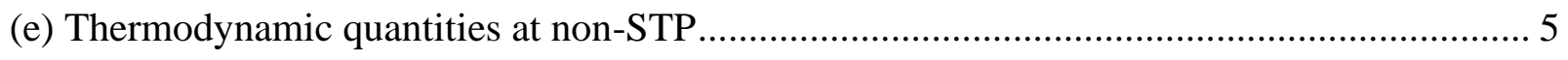

(f) Energy in the aqueous phase at non-STP (SMD model) ........................................... 5

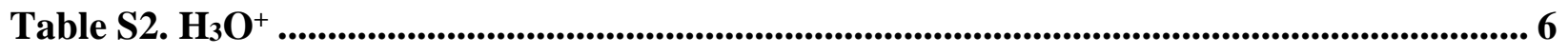

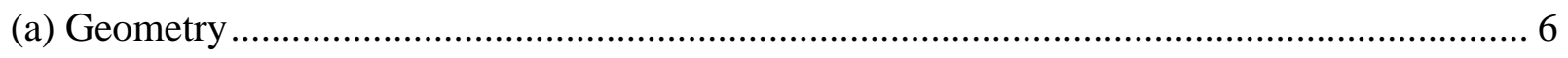

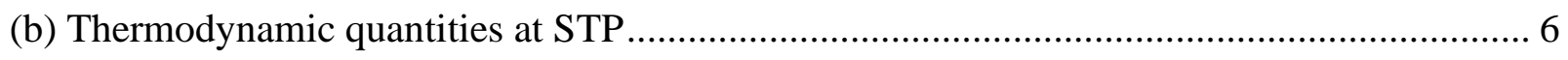

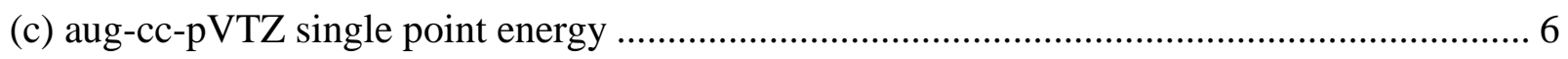

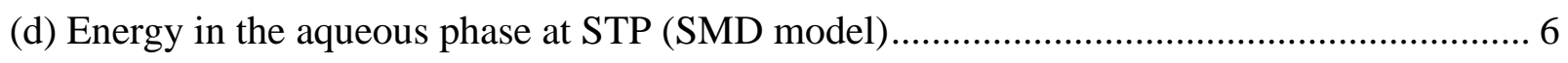

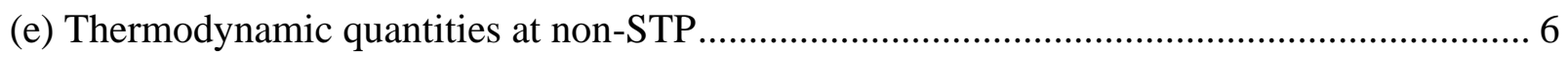

(f) Energy in the aqueous phase at non-STP (SMD model) ............................................... 6

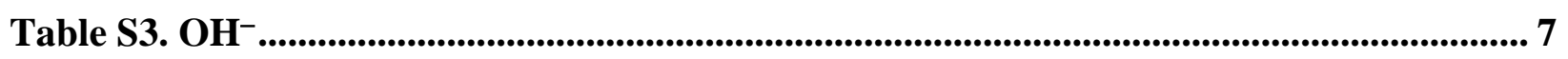

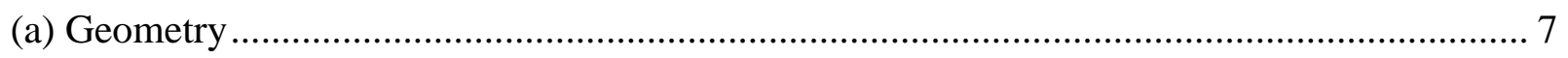

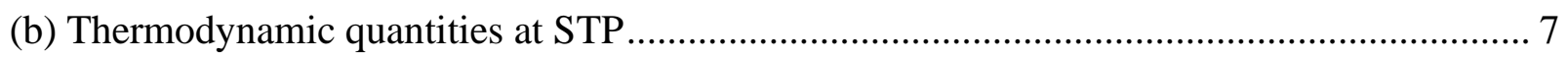

(c) aug-cc-pVTZ single point energy ............................................................................. 7

(d) Energy in the aqueous phase at STP (SMD model) .............................................. 7

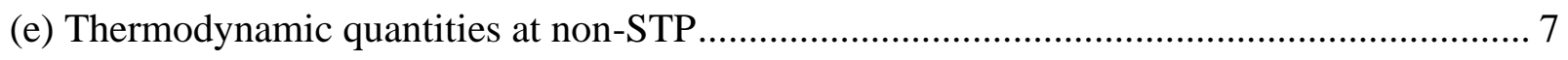

(f) Energy in the aqueous phase at non-STP (SMD model) …...................................... 7

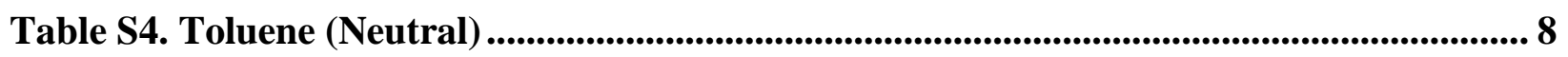

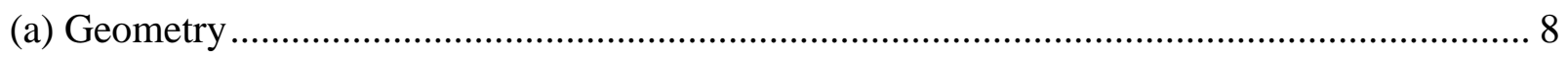

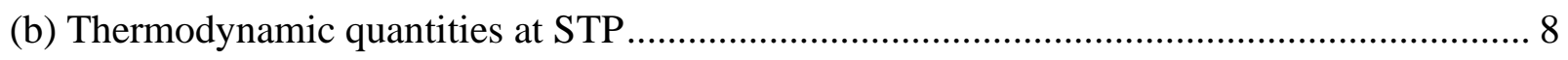




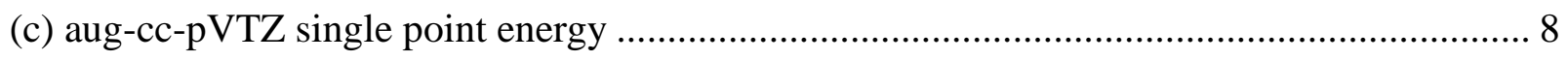

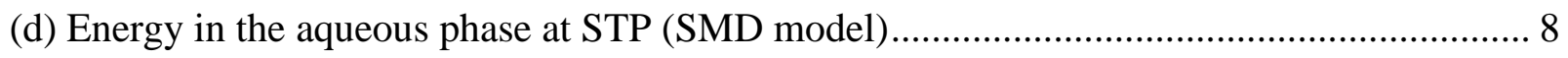

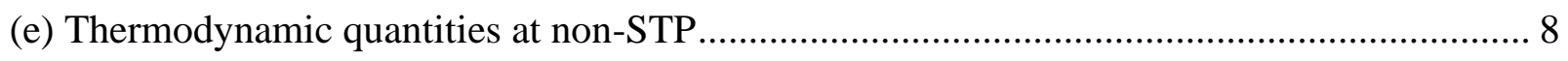

(f) Energy in the aqueous phase at non-STP (SMD model) ................................................... 9

Table S5. Toluene: $\alpha$-anion .......................................................................................................................... 9

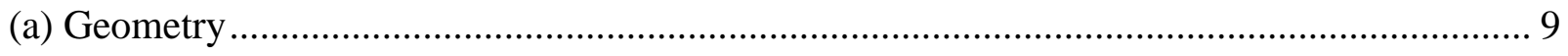

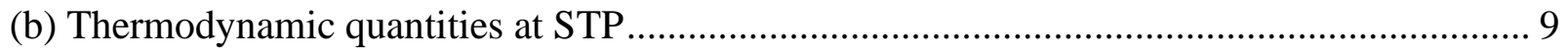

(c) aug-cc-pVTZ single point energy ……..................................................................... 9

(d) Energy in the aqueous phase at STP (SMD model) .......................................................... 9

(e) Thermodynamic quantities at non-STP......................................................................... 10

(f) Energy in the aqueous phase at non-STP (SMD model) ................................................... 10

Table S6. Toluene: ortho-anion ......................................................................................................... 10

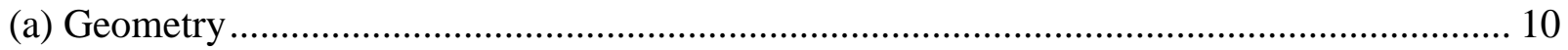

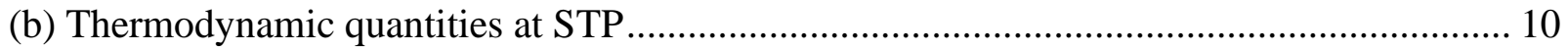

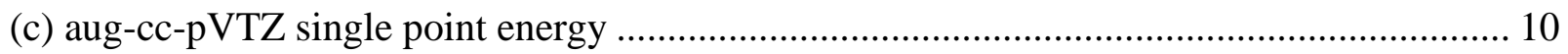

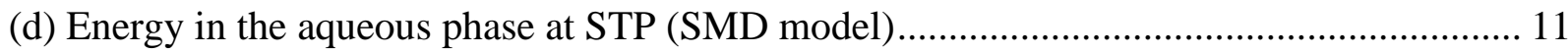

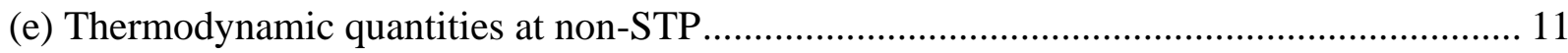

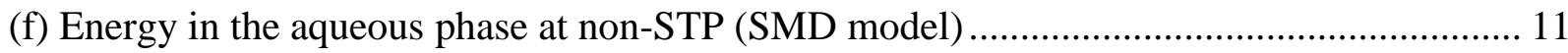

Table S7. Toluene: meta-anion ............................................................................................................. 11

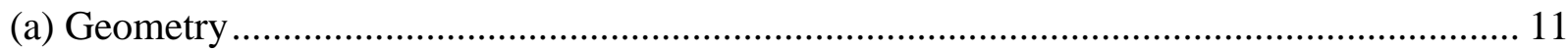

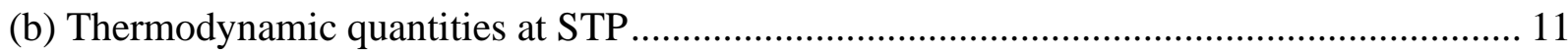

(c) aug-cc-pVTZ single point energy ............................................................................... 12

(d) Energy in the aqueous phase at STP (SMD model) ...................................................... 12

(e) Thermodynamic quantities at non-STP....................................................................... 12

(f) Energy in the aqueous phase at non-STP (SMD model) ………….................................. 12

Table S8. Toluene: para-anion......................................................................................................... 12

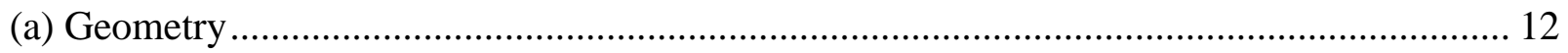

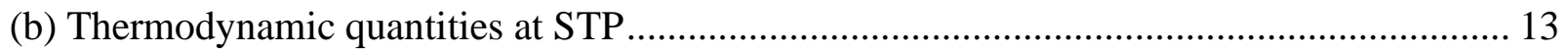

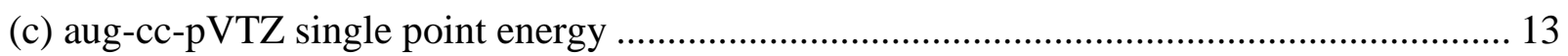

(d) Energy in the aqueous phase at STP (SMD model) ....................................................... 13

(e) Thermodynamic quantities at non-STP........................................................................ 13

(f) Energy in the aqueous phase at non-STP (SMD model) .................................................. 13

Table S9. n-Butylbenzene (neutral) ................................................................................................... 13

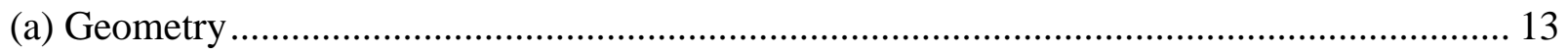




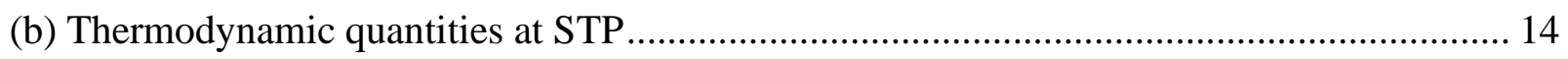

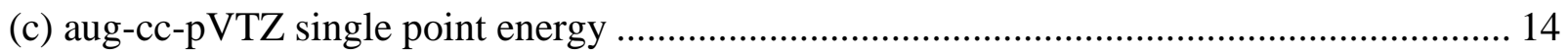

(d) Energy in the aqueous phase at STP (SMD model) ....................................................... 14

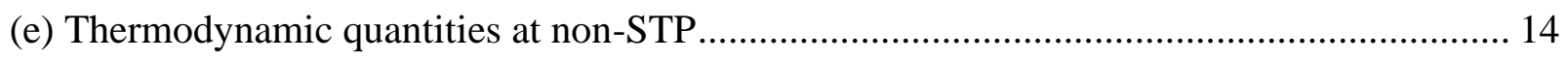

(f) Energy in the aqueous phase at non-STP (SMD model) .................................................... 14

Table S10. n-Butylbenzene: $\alpha$-anion .......................................................................................... 15

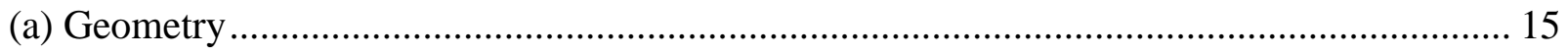

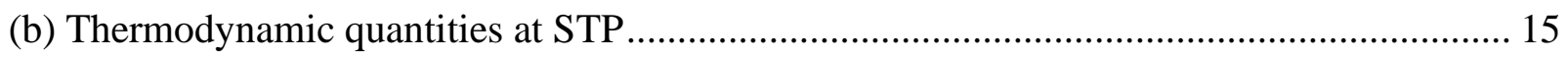

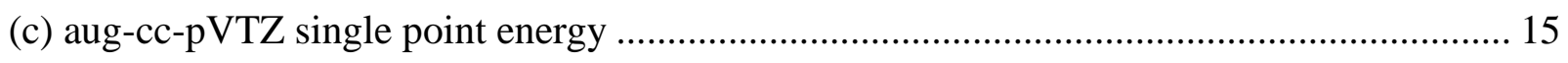

(d) Energy in the aqueous phase at STP (SMD model) ....................................................... 15

(e) Thermodynamic quantities at non-STP....................................................................... 16

(f) Energy in the aqueous phase at non-STP (SMD model) ................................................... 16

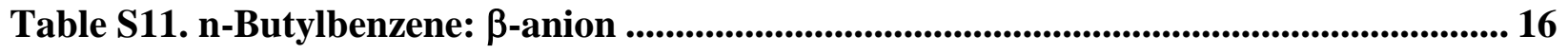

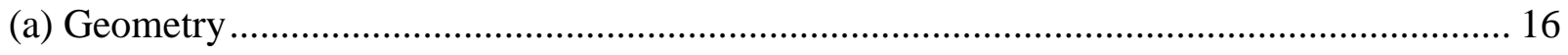

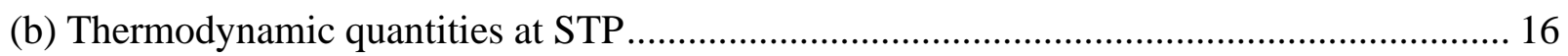

(c) aug-cc-pVTZ single point energy ……………........................................................... 17

(d) Energy in the aqueous phase at STP (SMD model) ........................................................ 17

(e) Thermodynamic quantities at non-STP....................................................................... 17

(f) Energy in the aqueous phase at non-STP (SMD model) ................................................... 17

Table S12. n-Butylbenzene: $\gamma$-anion ......................................................................................... 17

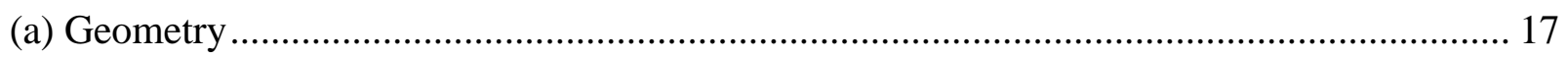

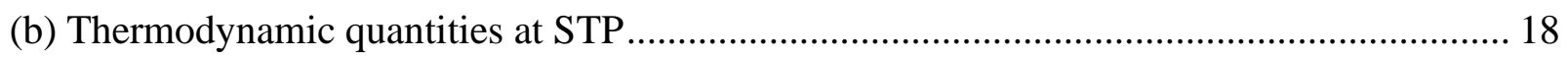

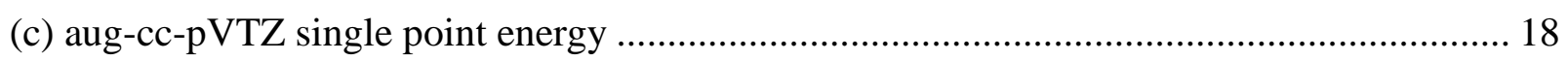

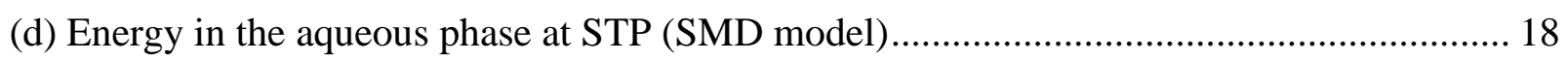

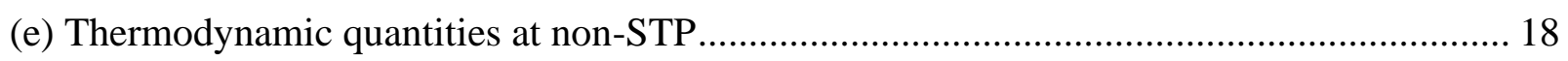

(f) Energy in the aqueous phase at non-STP (SMD model) ................................................. 18

Table S13. n-Butylbenzene: $\delta$-anion....................................................................................................... 18

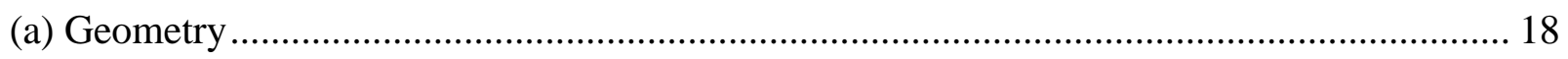

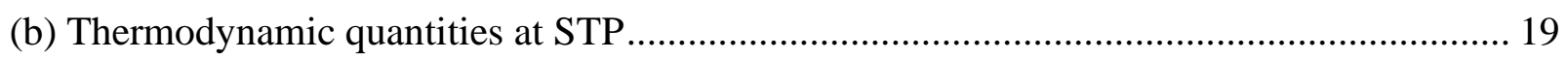

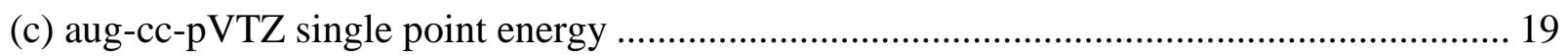

(d) Energy in the aqueous phase at STP (SMD model) ....................................................... 19

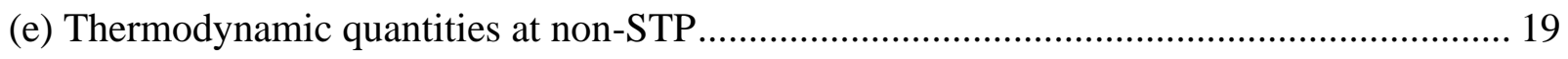

(f) Energy in the aqueous phase at non-STP (SMD model) ................................................ 20

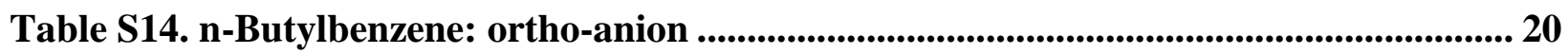




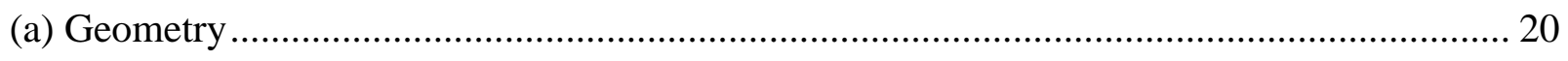

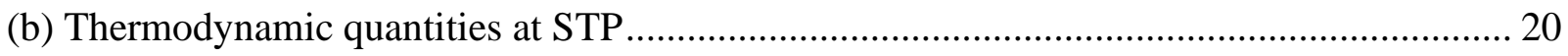

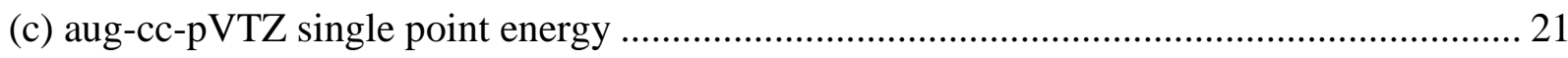

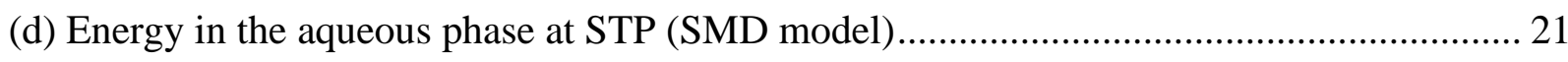

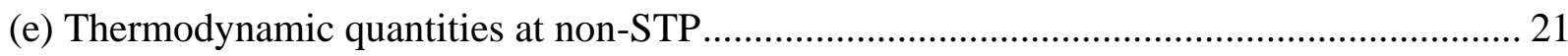

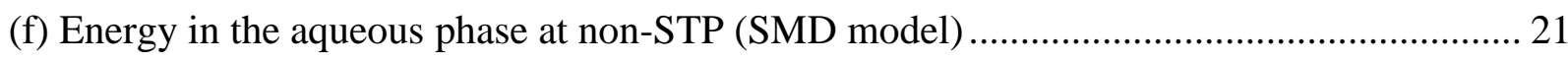

Table S15. n-Butylbenzene: meta-anion ............................................................................................... 21

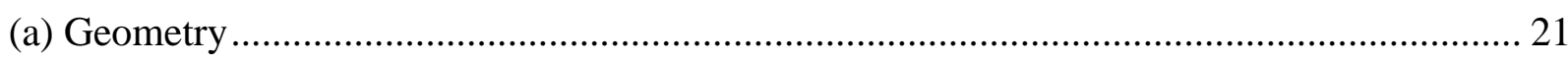

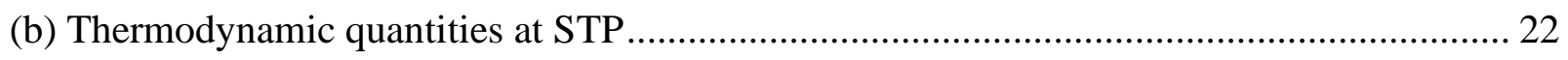

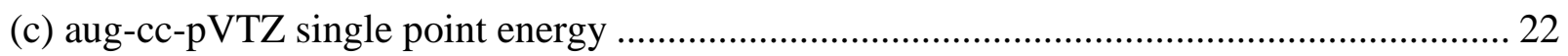

(d) Energy in the aqueous phase at STP (SMD model) ...................................................... 22

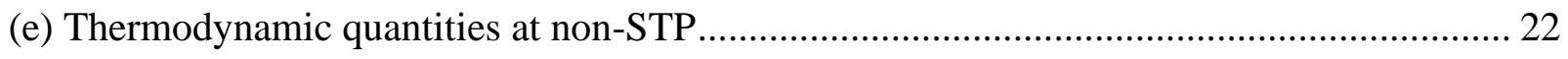

(f) Energy in the aqueous phase at non-STP (SMD model) ................................................. 22

Table S16. n-Butylbenzene: para-anion............................................................................................ 22

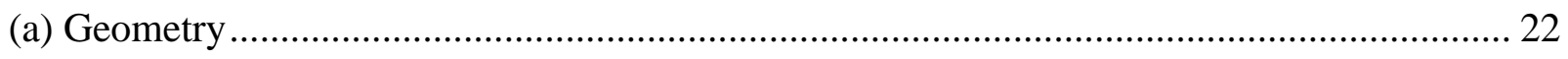

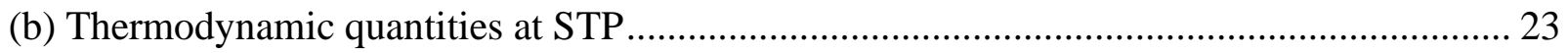

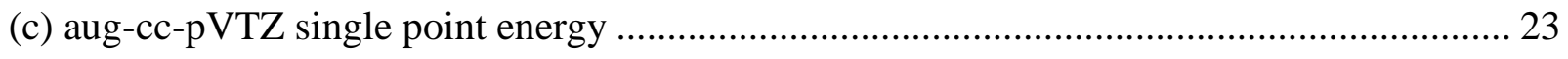

(d) Energy in the aqueous phase at STP (SMD model) ........................................................ 23

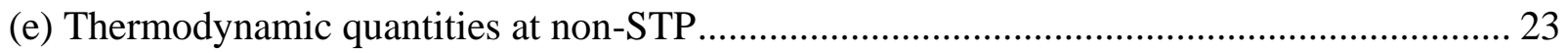

(f) Energy in the aqueous phase at non-STP (SMD model) .................................................. 24

Table S17. Relaxed PE Scans ............................................................................................................ 25

Figure S1. The relative energies from the relaxed PE scans of Table S17............................ 26

Figure S2. Additional stabilization of the ortho anion ....................................................................... 27

All calculations use M06-2X functional and the cc-pVTZ basis unless otherwise specified. All SMD calculations used $\varepsilon=78.3553$ at STP (G16 default) and 5.1000 for non-STP. 


\section{Table S1. $\mathrm{H}_{2} \mathrm{O}$}

\section{(a) Geometry}

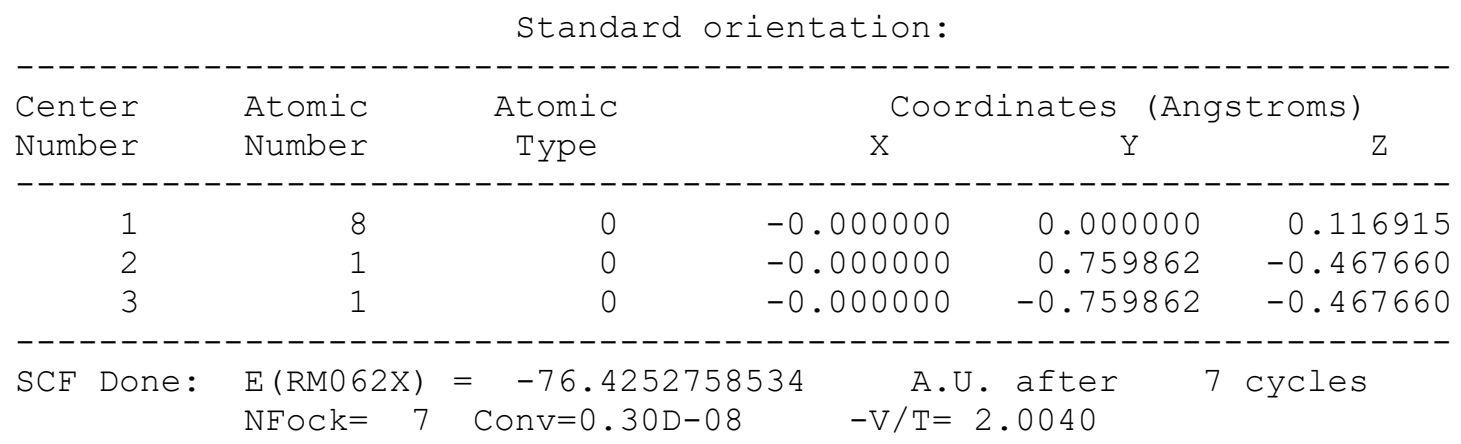

(b) Thermodynamic quantities at STP

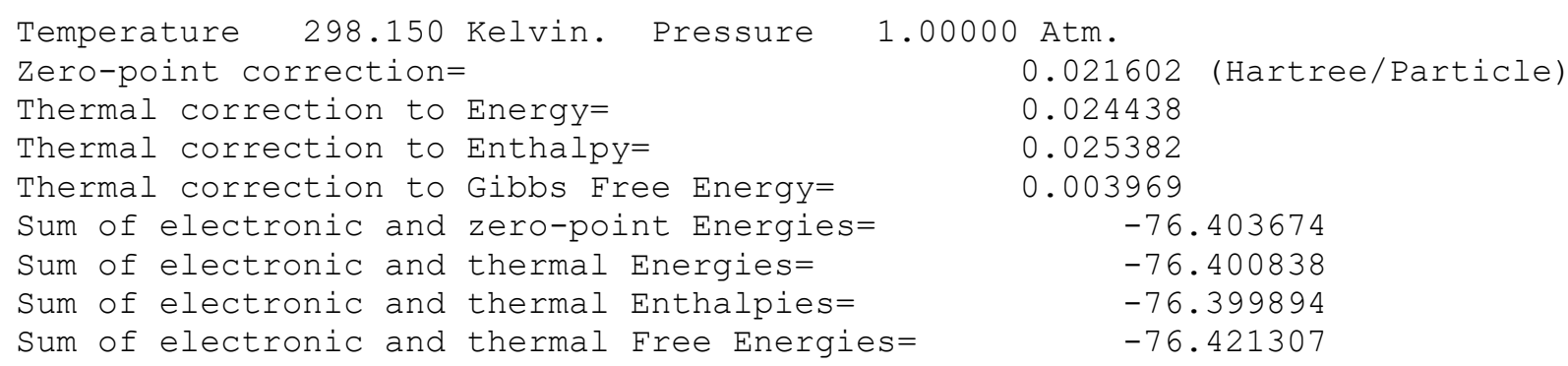

(c) aug-cc-pVTZ single point energy

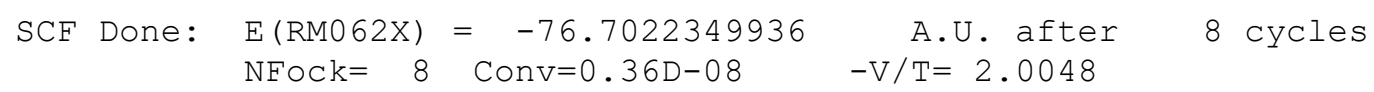

(d) Energy in the aqueous phase at STP (SMD model)

$\begin{array}{cccc}\mathrm{SCF} \text { Done: } \mathrm{E}(\mathrm{RM} 062 \mathrm{X})=-76.8539118485 & \mathrm{~A} . \mathrm{U} \text {. after } & 8 \text { cycles } \\ \mathrm{NFock}=8 \text { Conv=0.27D-08 } & -\mathrm{V} / \mathrm{T}=2.0070 & \\ \text { SMD-CDS (non-electrostatic) energy } & (\mathrm{kCal} / \mathrm{mol})= & 2.17 \\ \text { (included in total energy above) } & & \end{array}$

(e) Thermodynamic quantities at non-STP

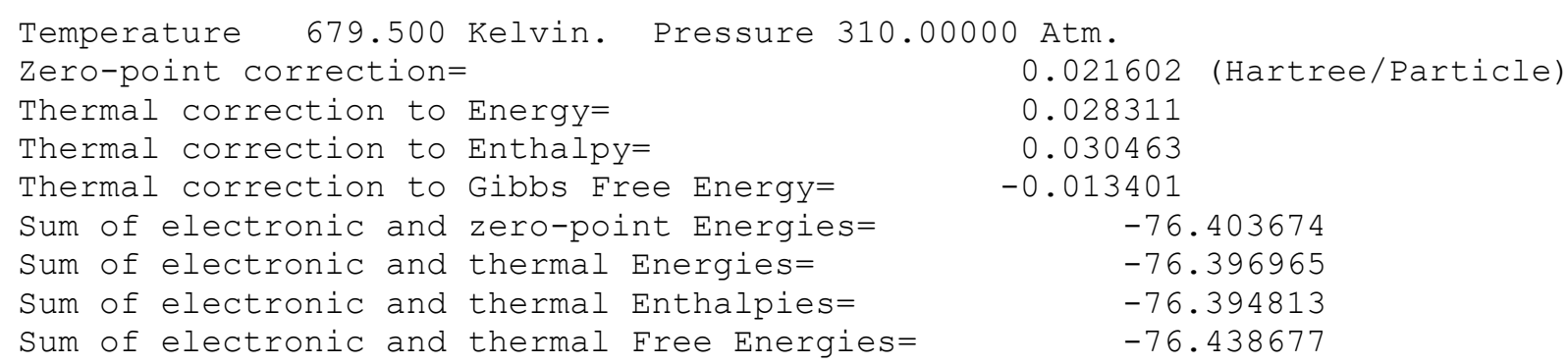

(f) Energy in the aqueous phase at non-STP (SMD model)

$\begin{array}{cccc}\mathrm{SCF} \text { Done: } & \mathrm{E}(\mathrm{RM} 062 \mathrm{X})=-76.4334486973 & \mathrm{~A} . \mathrm{U} . \text { after } & 8 \text { cycles } \\ \mathrm{NFock}=8 \text { Conv=0.26D-08 } & -\mathrm{V} / \mathrm{T}=2.0042 & \\ \text { SMD-CDS (non-electrostatic) energy } & (\mathrm{kcal} / \mathrm{mol})= & 1.42 \\ \text { (included in total energy above) } & & \end{array}$




\section{Table S2. $\mathrm{H}_{3} \mathrm{O}^{+}$}

\section{(a) Geometry}

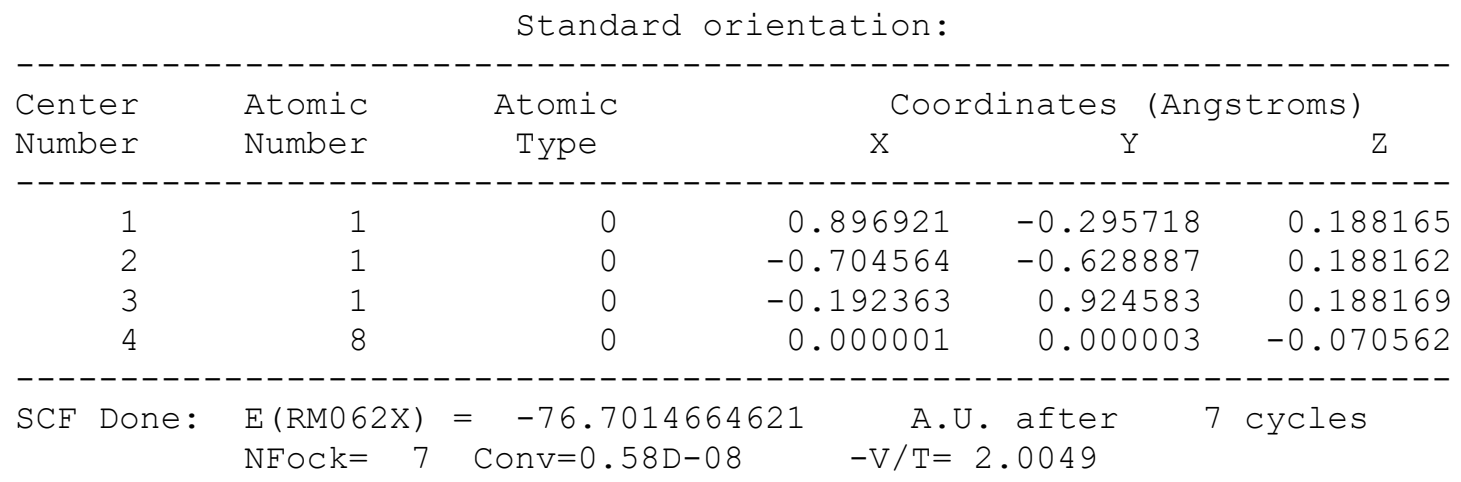

(b) Thermodynamic quantities at STP

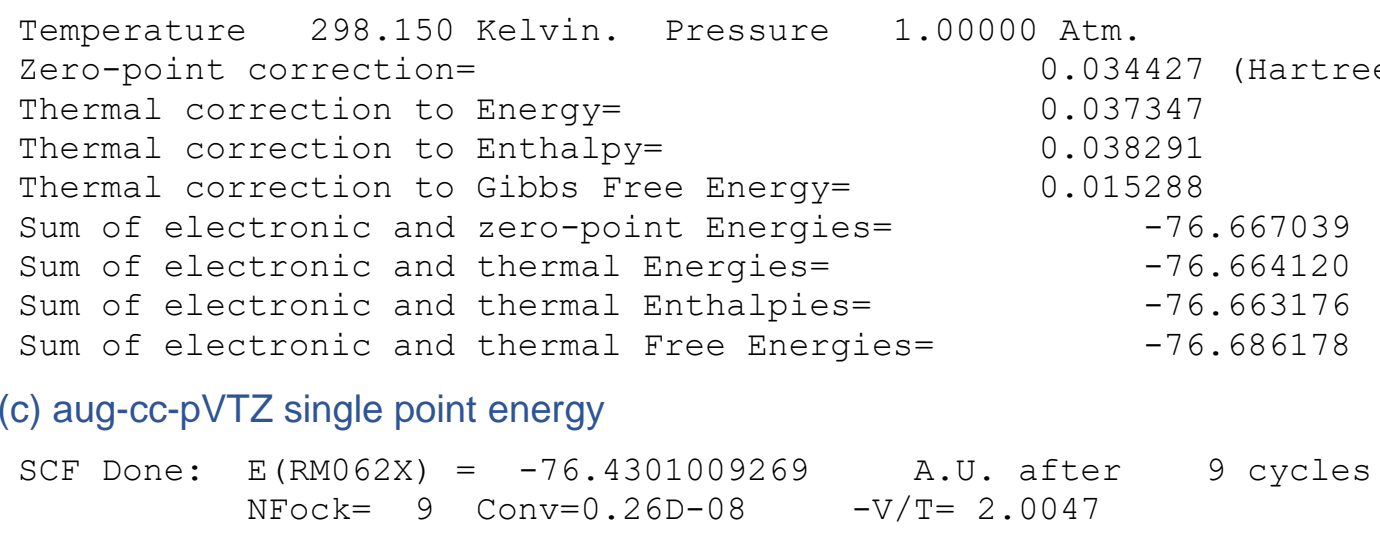

(d) Energy in the aqueous phase at STP (SMD model)

$\begin{array}{ccccc}\mathrm{SCF} \text { Done: } & \mathrm{E}(\mathrm{RM} 06 \mathrm{X})=-76.4374178251 & \begin{array}{c}\text { A.U. after } \\ \text { NFock= } 8 \text { Conv=0.52D-08 }\end{array} & -\mathrm{V} / \mathrm{T}=2.0043 & \text { cycles } \\ \text { SMD-CDS (non-electrostatic) energy } & (\mathrm{kcal} / \mathrm{mol})= & 1.42\end{array}$

(included in total energy above)

(e) Thermodynamic quantities at non-STP

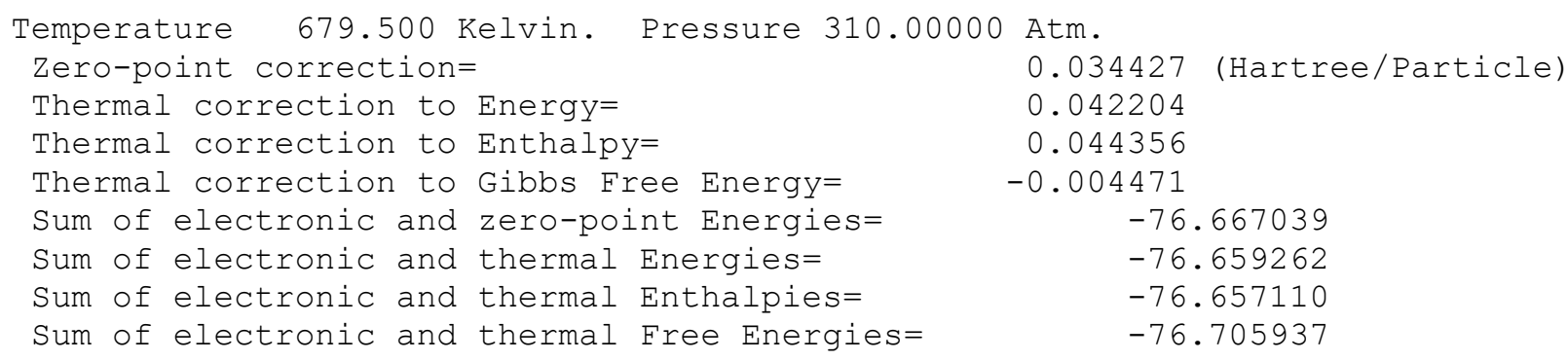

(f) Energy in the aqueous phase at non-STP (SMD model)

$\begin{array}{ccccc}\mathrm{SCF} \text { Done: } & \mathrm{E}(\mathrm{RM} 06 \mathrm{X})=-76.8247536713 & \begin{array}{c}\mathrm{A} . \mathrm{U} \text {. after } \\ \text { NFock= } 8 \text { Conv=0.19D-08 }\end{array} & \begin{array}{c}\mathrm{V} / \mathrm{T}=2.0066 \\ \text { cycles }\end{array} \\ \text { SMD-CDS (non-electrostatic) energy } & (\mathrm{kcal} / \mathrm{mol})= & 2.17 \\ \text { (included in total energy above) } & & \end{array}$




\section{Table S3. $\mathrm{OH}^{-}$}

(a) Geometry

Standard orientation:

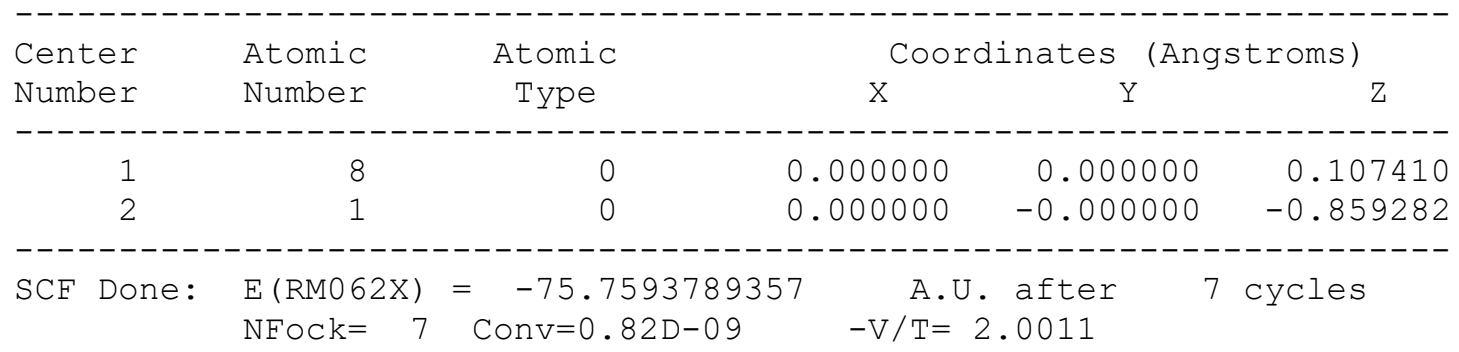

(b) Thermodynamic quantities at STP

$\begin{array}{lcc}\text { Temperature } 298.150 \text { Kelvin. } & \text { Pressure } & 1.00000 \text { Atm. } \\ \text { Zero-point correction= } & 0.008401 & \text { (Hartree/Particle) } \\ \text { Thermal correction to Energy= } & 0.010761 & 0.011705 \\ \text { Thermal correction to Enthalpy= } & -0.007855 \\ \text { Thermal correction to Gibbs Free Energy= } & -75.750978 \\ \text { Sum of electronic and zero-point Energies= } & -75.748618 \\ \text { Sum of electronic and thermal Energies= } & -75.747673 \\ \text { Sum of electronic and thermal Enthalpies= } & -75.767234 \\ \text { Sum of electronic and thermal Free Energies= } & \end{array}$

(c) aug-cc-pVTZ single point energy

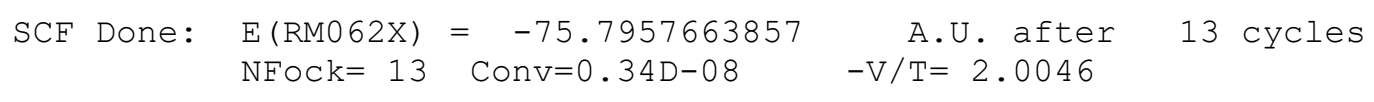

(d) Energy in the aqueous phase at STP (SMD model)

$\begin{array}{cccc}\mathrm{SCF} \text { Done: } \mathrm{E}(\mathrm{RM} 062 \mathrm{X})=-75.9259242397 & \begin{array}{c}\mathrm{A} . \mathrm{U} . \text { after } \\ \text { NFock= 9 Conv=0.29D-08 }\end{array} & \begin{array}{c}\mathrm{V} / \mathrm{T}=2.0032 \\ \text { cycles }\end{array} \\ \text { SMD-CDS (non-electrostatic) energy } & (\mathrm{kcal} / \mathrm{mol})= & 0.74 \\ \text { (included in total energy above) } & & \end{array}$

(e) Thermodynamic quantities at non-STP

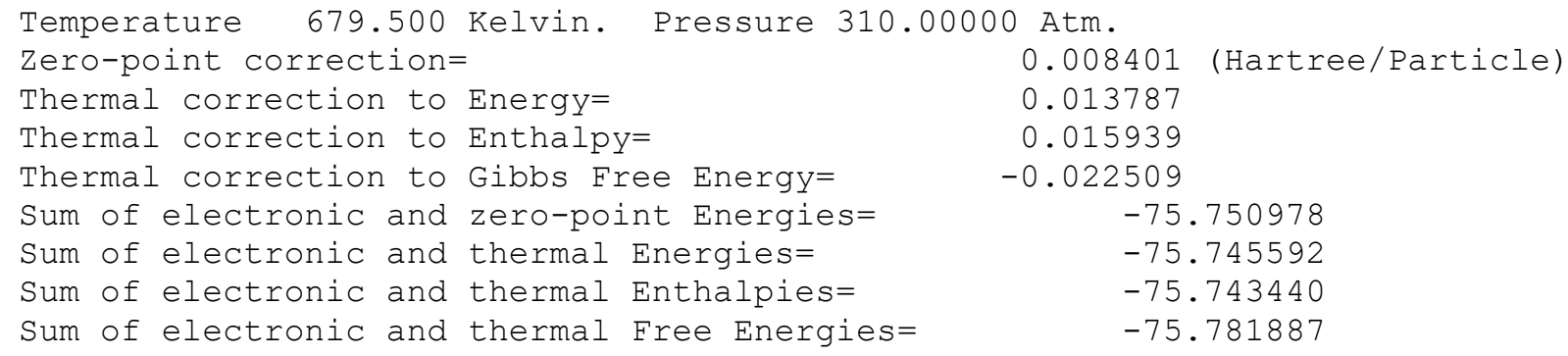

(f) Energy in the aqueous phase at non-STP (SMD model)

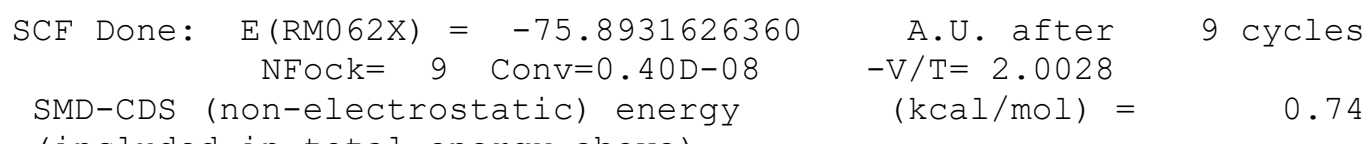




\section{Table S4. Toluene (Neutral)}

\section{(a) Geometry}

\begin{tabular}{|c|c|c|c|c|c|}
\hline \multirow{2}{*}{$\begin{array}{l}\text { Center } \\
\text { Number }\end{array}$} & \multirow{2}{*}{$\begin{array}{l}\text { Atomic } \\
\text { Number }\end{array}$} & \multirow{2}{*}{$\begin{array}{c}\text { Atomic } \\
\text { Type }\end{array}$} & \multicolumn{3}{|c|}{ Coordinates (Angstroms) } \\
\hline & & & $\mathrm{X}$ & $\mathrm{Y}$ & Z \\
\hline \multicolumn{6}{|l|}{------1} \\
\hline 1 & 6 & 0 & 1.193362 & -1.198364 & 0.001995 \\
\hline 2 & 6 & 0 & -0.193797 & -1.195183 & -0.008651 \\
\hline 3 & 6 & 0 & -0.907309 & -0.000000 & -0.011287 \\
\hline 4 & 6 & 0 & -0.193797 & 1.195183 & -0.008651 \\
\hline 5 & 6 & 0 & 1.193362 & 1.198364 & 0.001995 \\
\hline 6 & 6 & 0 & 1.892657 & 0.000000 & 0.008278 \\
\hline 7 & 1 & 0 & 1.728991 & -2.138273 & 0.001675 \\
\hline 8 & 1 & 0 & -0.733651 & -2.134336 & -0.017849 \\
\hline 9 & 1 & 0 & -0.733651 & 2.134336 & -0.017849 \\
\hline 10 & 1 & 0 & 1.728991 & 2.138273 & 0.001675 \\
\hline 11 & 1 & 0 & 2.974057 & 0.000000 & 0.013648 \\
\hline 12 & 6 & 0 & -2.410935 & 0.000000 & 0.009033 \\
\hline 13 & 1 & 0 & -2.811489 & -0.883274 & -0.486429 \\
\hline 14 & 1 & 0 & -2.783011 & 0.000000 & 1.035290 \\
\hline 15 & 1 & 0 & -2.811489 & 0.883274 & -0.486429 \\
\hline \multicolumn{6}{|c|}{ 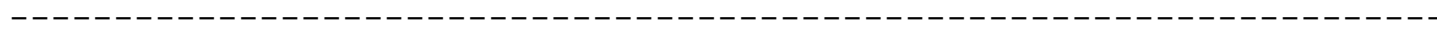 } \\
\hline \multirow[t]{2}{*}{ SCF Done: } & $\mathrm{E}(\mathrm{RM} 062 \mathrm{X})$ & $=-271$ & \multirow{2}{*}{\multicolumn{2}{|c|}{$\begin{array}{l}\text { A.U. after } \\
-\mathrm{V} / \mathrm{T}=2.0060\end{array}$}} & cycles \\
\hline & $\mathrm{NFOCk}=$ & $\mathrm{Co}$ & & & \\
\hline
\end{tabular}

(b) Thermodynamic quantities at STP

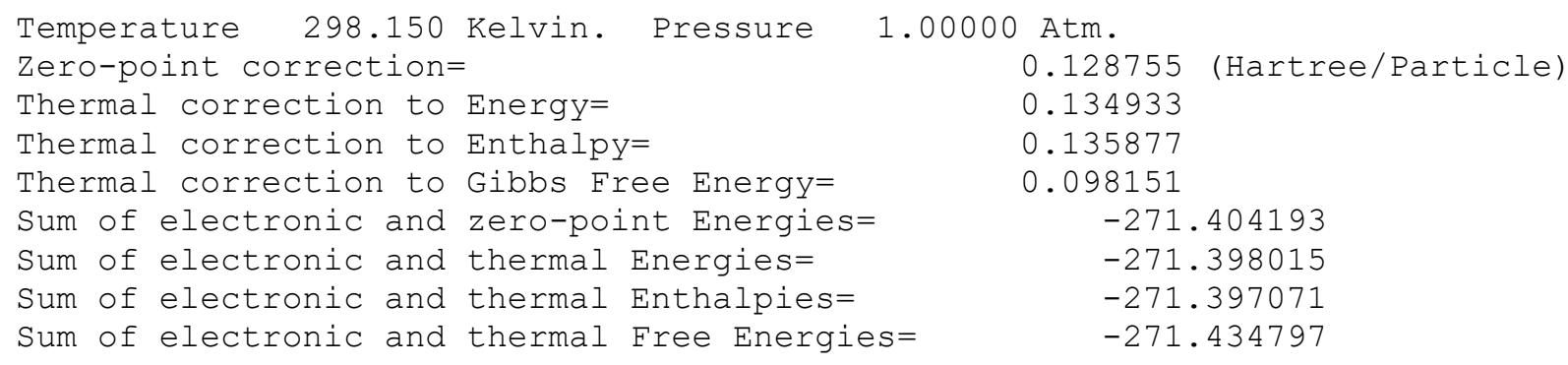

(c) aug-cc-pVTZ single point energy

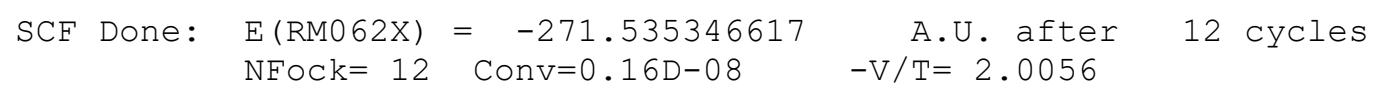

(d) Energy in the aqueous phase at STP (SMD model)

$\begin{array}{llrr}\mathrm{SCF} \text { Done: } & \mathrm{E}(\mathrm{RM} 062 \mathrm{X})=-271.534308266 & \mathrm{~A} . \mathrm{U} . \text { after } & 11 \text { cycles } \\ \mathrm{NFOCk}=11 \text { Conv=0.53D-08 } & -\mathrm{V} / \mathrm{T}=2.0060\end{array}$

(e) Thermodynamic quantities at non-STP

$\begin{array}{lcc}\text { Temperature } 679.500 \text { Kelvin. } & \text { Pressure } 310.00000 \text { Atm. } \\ \text { Zero-point correction= } & 0.128755 & \text { (Hartree/Particle) } \\ \text { Thermal correction to Energy= } & 0.157587 & \\ \text { Thermal correction to Enthalpy= } & 0.159739 \\ \text { Thermal correction to Gibbs Free Energy= } & 0.052677 \\ \text { Sum of electronic and zero-point Energies= } & -271.404193 \\ \text { Sum of electronic and thermal Energies= } & -271.375361\end{array}$


Sum of electronic and thermal Enthalpies=

$-271.373209$

Sum of electronic and thermal Free Energies=

$-271.480271$

(f) Energy in the aqueous phase at non-STP (SMD model)

$\begin{array}{cccc}\mathrm{SCF} \text { Done: } \mathrm{E}(\mathrm{RM} 062 \mathrm{X})=-271.532331077 & \mathrm{~A} . \mathrm{U} \text {. after } & 11 \text { cycles } \\ \mathrm{NFOCk}=11 \quad \text { Conv=0.39D-08 } & -\mathrm{V} / \mathrm{T}=2.0060 & \\ \text { SMD-CDS (non-electrostatic) energy } & (\mathrm{kcal} / \mathrm{mol})= & 3.20 \\ \text { (included in total energy above) } & \end{array}$

\section{Table S5. Toluene: $\alpha$-anion}

(a) Geometry

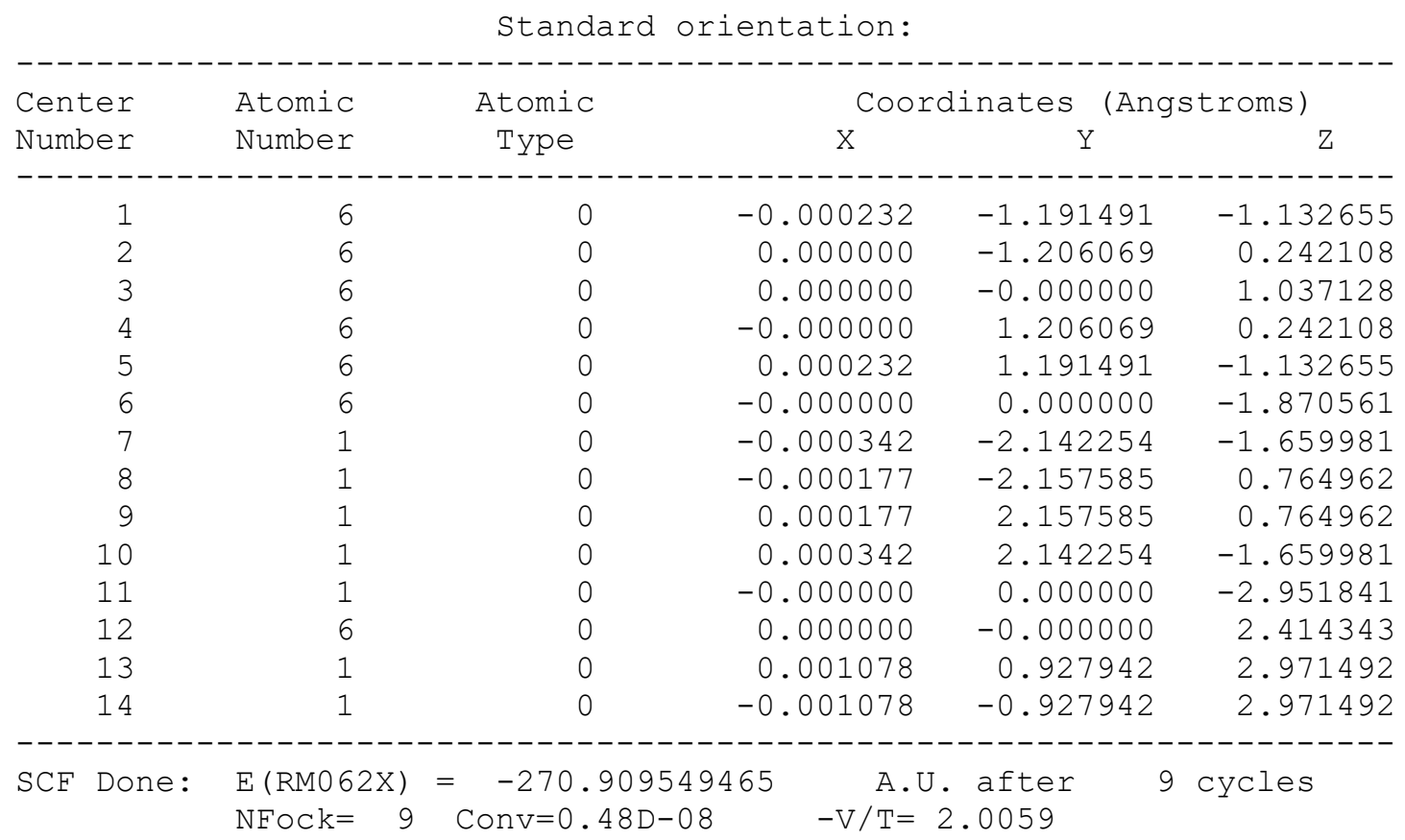

(b) Thermodynamic quantities at STP

\begin{tabular}{|c|c|c|}
\hline $\begin{array}{l}\text { Temperature } 298.150 \\
\text { Zero-point correction= }\end{array}$ & Kelvin. Pressure & $\begin{array}{l}\text { Atm. } \\
0.113065 \text { (Hartree/Particle) }\end{array}$ \\
\hline Thermal correction to & Energy $=$ & 0.118950 \\
\hline Thermal correction to & Enthalpy $=$ & 0.119894 \\
\hline Thermal correction to & Gibbs Free Energy= & 0.084550 \\
\hline Sum of electronic and & zero-point Energies= & -270.796485 \\
\hline Sum of electronic and & thermal Energies $=$ & -270.790600 \\
\hline Sum of electronic and & thermal Enthalpies= & -270.789656 \\
\hline Sum of electronic and & thermal Free Energies= & -270.824999 \\
\hline
\end{tabular}

(c) aug-cc-pVTZ single point energy

$$
\begin{array}{llll}
\mathrm{SCF} \text { Done: } & \mathrm{E}(\mathrm{RM} 062 \mathrm{X})=-270.917522480 & \text { A.U. after } & 12 \text { cycles } \\
& \text { NFock=12 Conv=0.52D-08 } & -\mathrm{V} / \mathrm{T}=2.0056 &
\end{array}
$$

(d) Energy in the aqueous phase at STP (SMD model)

$\begin{array}{cccc}\mathrm{SCF} \text { Done: } \quad \mathrm{E}(\mathrm{RM} 062 \mathrm{X})=-270.995989367 & \begin{array}{c}\mathrm{A} . \mathrm{U} . \text { after } \\ \mathrm{NFOCk}=13 \quad \text { Conv=0.74D-08 }\end{array} & -\mathrm{V} / \mathrm{T}=2.0059 & \\ \text { SMD-CDS (non-electrostatic) energy } & (\mathrm{kcal} / \mathrm{mol})= & 3.69 \\ \text { (included in total energy above) } & & \end{array}$


(e) Thermodynamic quantities at non-STP

$\begin{array}{ll}\text { Temperature } 679.500 \text { Kelvin. } & \text { Pressure } 310.00000 \\ \text { Zero-point correction= } & 0.113065 \text { (Hartree/Particle) } \\ \text { Thermal correction to Energy= } & 0.141659 \\ \text { Thermal correction to Enthalpy= } & 0.143811 \\ \text { Thermal correction to Gibbs Free Energy= } & 0.041971 \\ \text { Sum of electronic and zero-point Energies= } & -270.796485 \\ \text { Sum of electronic and thermal Energies= } & -270.767891 \\ \text { Sum of electronic and thermal Enthalpies= } & -270.765739 \\ \text { Sum of electronic and thermal Free Energies= } & -270.867579\end{array}$

(f) Energy in the aqueous phase at non-STP (SMD model)

$\begin{array}{ccccc}\mathrm{SCF} \text { Done: } & \mathrm{E}(\mathrm{RM} 06 \mathrm{X})= & -270.976568869 & \mathrm{~A} . \mathrm{U} \text {. after } & 13 \text { cycles } \\ \mathrm{NFOCk}=13 & \mathrm{Conv}=0.51 \mathrm{D}-08 & -\mathrm{V} / \mathrm{T}=2.0059 & \\ \text { SMD-CDS (non-electrostatic) energy } & (\mathrm{kcal} / \mathrm{mol})= & 3.69\end{array}$

Table S6. Toluene: ortho-anion

(a) Geometry

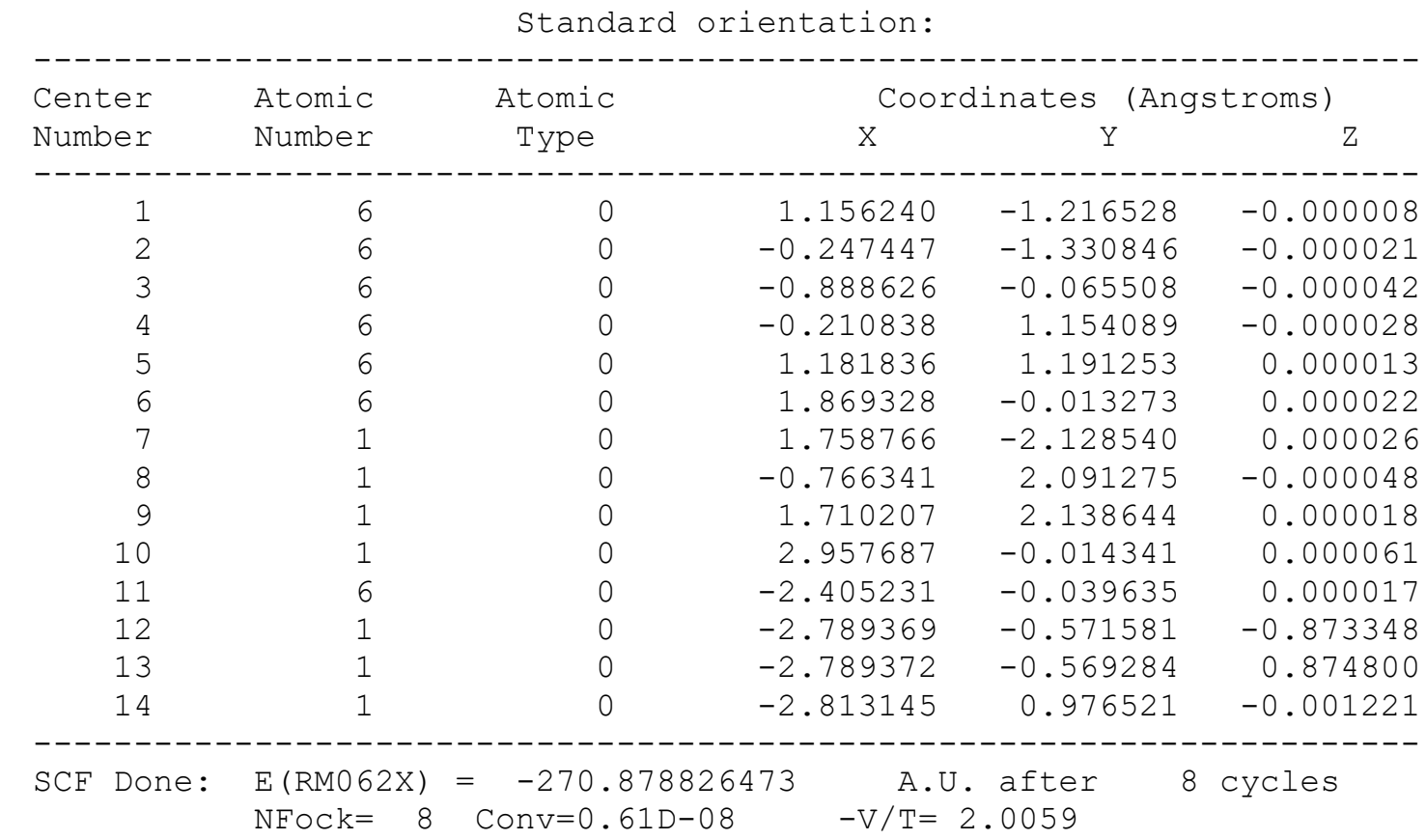

(b) Thermodynamic quantities at STP

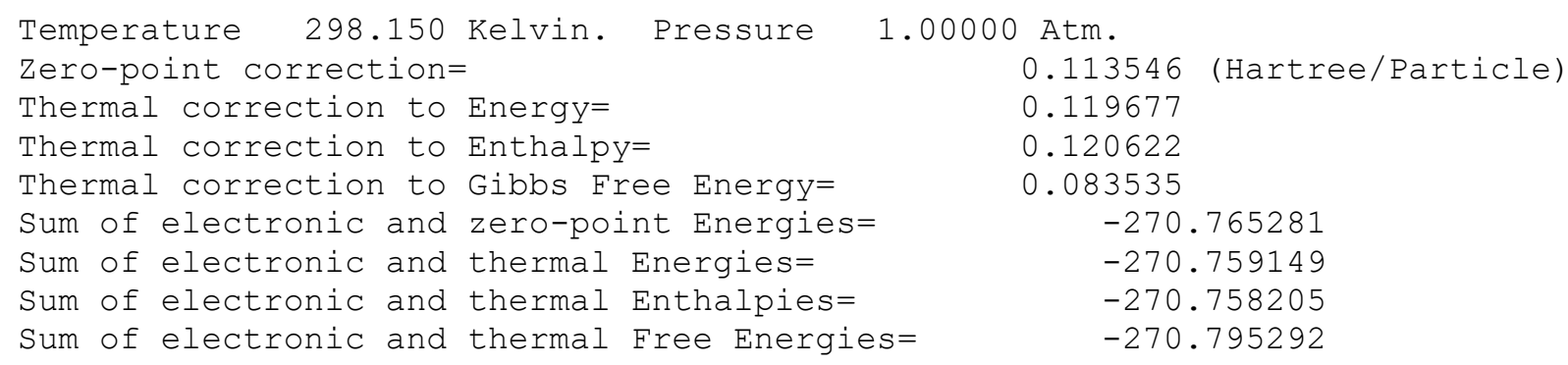

(c) aug-cc-pVTZ single point energy

$\begin{array}{llrr}\mathrm{SCF} \text { Done: } & \mathrm{E}(\mathrm{RM} 062 \mathrm{X})=-270.887374418 & \text { A.U. after } & 14 \text { cycles } \\ & \mathrm{NFOCk}=14 \quad \mathrm{Conv}=0.38 \mathrm{D}-08 & -\mathrm{V} / \mathrm{T}=2.0056\end{array}$


(d) Energy in the aqueous phase at STP (SMD model)

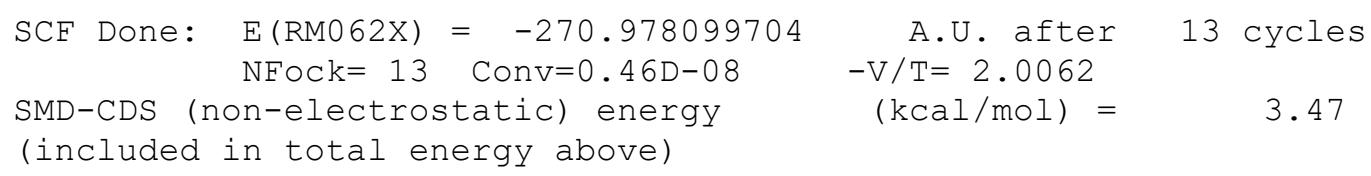

(e) Thermodynamic quantities at non-STP

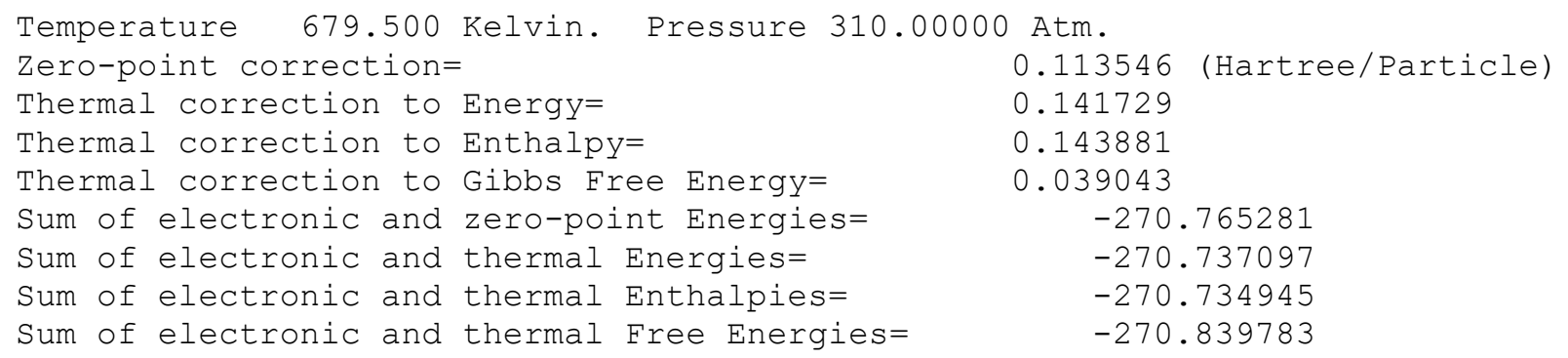

(f) Energy in the aqueous phase at non-STP (SMD model)

$\begin{array}{cccc}\mathrm{SCF} \text { Done: } \mathrm{E}(\mathrm{RM} 062 \mathrm{X})=-270.954840023 & \mathrm{~A} . \mathrm{U} \text {. after } & 13 \text { cycles } \\ \mathrm{NFock}=13 \quad \text { Conv=0.92D-08 } & -\mathrm{V} / \mathrm{T}=2.0061 & \\ \text { SMD-CDS (non-electrostatic) energy } & (\mathrm{kcal} / \mathrm{mol})= & 3.47 \\ \text { (included in total energy above) } & \end{array}$

\section{Table S7. Toluene: meta-anion}

(a) Geometry

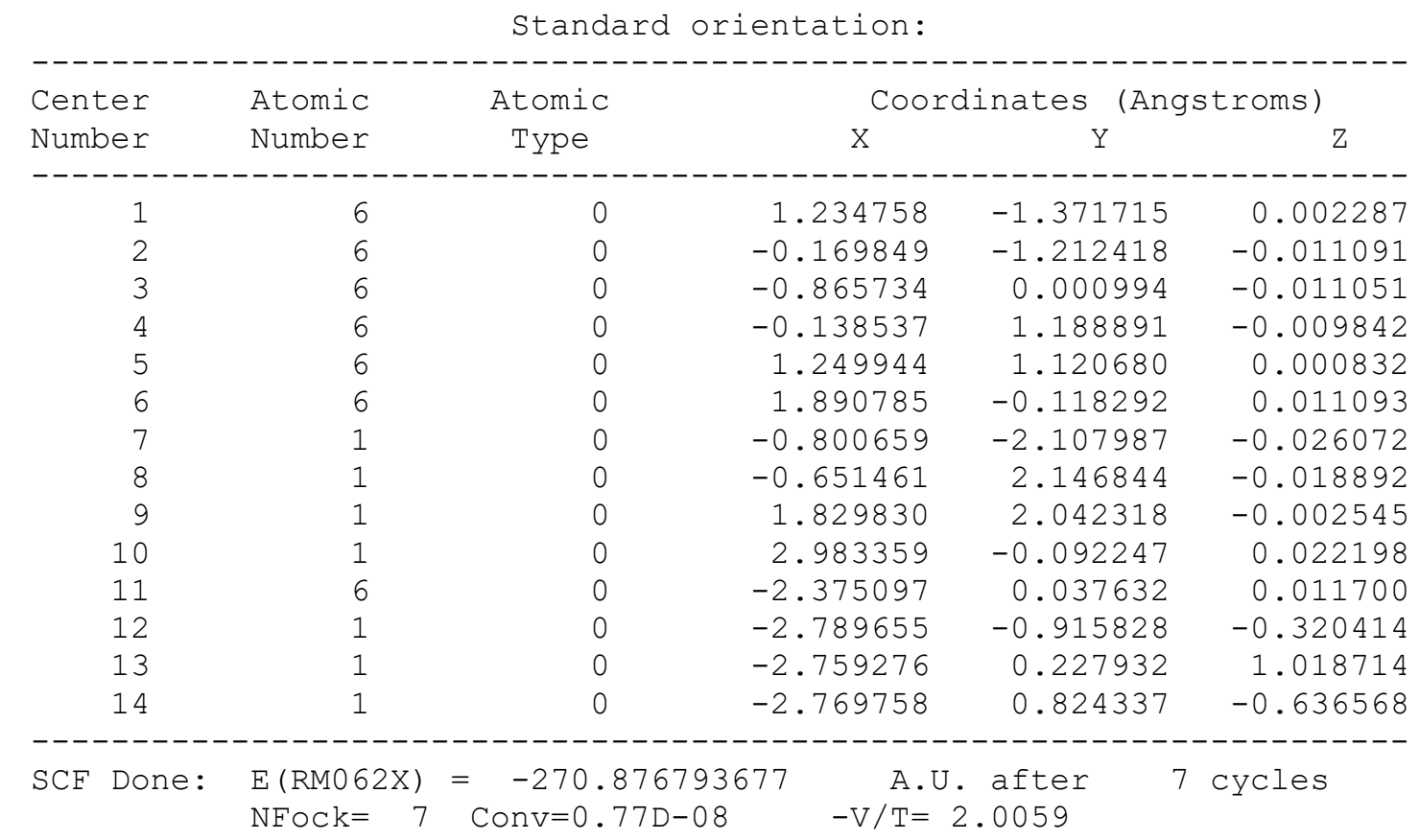

(b) Thermodynamic quantities at STP

$\begin{array}{lcc}\text { Temperature } 298.150 \text { Kelvin. } & \text { Pressure } & 1.00000 \text { Atm. } \\ \text { Zero-point correction= } & 0.113523 & \text { (Hartree/Particle) } \\ \text { Thermal correction to Energy= } & 0.119711 \\ \text { Thermal correction to Enthalpy= } & 0.120655 \\ \text { Thermal correction to Gibbs Free Energy= } & 0.083182 \\ \text { Sum of electronic and zero-point Energies= } & -270.763271\end{array}$


Sum of electronic and thermal Energies=

$-270.757083$

Sum of electronic and thermal Enthalpies=

$-270.756139$

Sum of electronic and thermal Free Energies=

$-270.793611$

(c) aug-cc-pVTZ single point energy

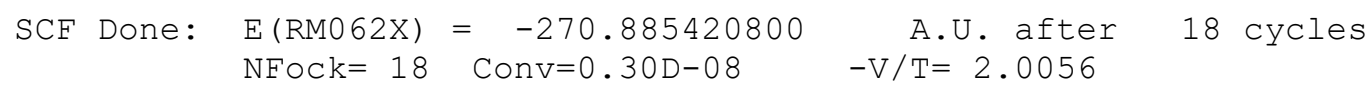

(d) Energy in the aqueous phase at STP (SMD model)

$\begin{array}{cccc}\mathrm{SCF} \text { Done: } & \mathrm{E}(\mathrm{RM} 062 \mathrm{X})=-270.978342511 & \mathrm{~A} . \mathrm{U} \text {. after } & 13 \text { cycles } \\ \mathrm{NFOCk}=13 & \mathrm{Conv}=0.89 \mathrm{D}-08 & -\mathrm{V} / \mathrm{T}=2.0062 & \\ \text { SMD-CDS (non-electrostatic) energy } & (\mathrm{kcal} / \mathrm{mol})= & 3.48\end{array}$

(included in total energy above)

(e) Thermodynamic quantities at non-STP

$\begin{array}{lcc}\text { Temperature } 679.500 \text { Kelvin. } & \text { Pressure } 310.00000 \text { Atm. } & \text { (Hartree/Particle) } \\ \text { Zero-point correction= } & 0.113529 & 0.141690 \\ \text { Thermal correction to Energy= } & 0.143842 \\ \text { Thermal correction to Enthalpy= } & 0.038367 \\ \text { Thermal correction to Gibbs Free Energy= } & -270.763265 \\ \text { Sum of electronic and zero-point Energies= } & -270.735104 \\ \text { Sum of electronic and thermal Energies= } & -270.732952 \\ \text { Sum of electronic and thermal Enthalpies= } & -270.838427 \\ \text { Sum of electronic and thermal Eree Energies= } & \end{array}$

(f) Energy in the aqueous phase at non-STP (SMD model)

$\begin{array}{cccc}\mathrm{SCF} \text { Done: } \mathrm{E}(\mathrm{RM} 062 \mathrm{X})=-270.954658204 & \mathrm{~A} . \mathrm{U} \text {. after } & 14 \text { cycles } \\ \mathrm{NFOCk}=14 \text { Conv=0.65D-08 } & -\mathrm{V} / \mathrm{T}=2.0061 & \\ \text { SMD-CDS (non-electrostatic) energy } & (\mathrm{kcal} / \mathrm{mol})= & 3.48 \\ \text { (included in total energy above) } & \end{array}$

Table S8. Toluene: para-anion

(a) Geometry

\begin{tabular}{|c|c|c|c|c|c|}
\hline \multirow{2}{*}{$\begin{array}{l}\text { Center } \\
\text { Number }\end{array}$} & \multirow{2}{*}{$\begin{array}{l}\text { Atomic } \\
\text { Number }\end{array}$} & \multirow{2}{*}{$\begin{array}{c}\text { Atomic } \\
\text { Type }\end{array}$} & \multicolumn{3}{|c|}{ Coordinates (Angstroms) } \\
\hline & & & $\mathrm{X}$ & Y & Z \\
\hline 1 & 6 & 0 & 1.254808 & -1.163528 & 0.003430 \\
\hline 2 & 6 & 0 & -0.141334 & -1.185173 & -0.009719 \\
\hline 3 & 6 & 0 & -0.871422 & -0.000000 & -0.013290 \\
\hline 4 & 6 & 0 & -0.141334 & 1.185173 & -0.009719 \\
\hline 5 & 6 & 0 & 1.254808 & 1.163528 & 0.003430 \\
\hline 6 & 6 & 0 & 2.058868 & 0.000000 & 0.011308 \\
\hline 7 & 1 & 0 & 1.742393 & -2.142417 & 0.001532 \\
\hline 8 & 1 & 0 & -0.679617 & -2.133929 & -0.020582 \\
\hline 9 & 1 & 0 & -0.679617 & 2.133929 & -0.020582 \\
\hline 10 & 1 & 0 & 1.742393 & 2.142417 & 0.001531 \\
\hline 11 & 6 & 0 & -2.379088 & 0.000000 & 0.011547 \\
\hline 12 & 1 & 0 & -2.782515 & -0.883430 & -0.488614 \\
\hline 13 & 1 & 0 & -2.772356 & 0.000000 & 1.033399 \\
\hline 14 & 1 & 0 & -2.782515 & 0.883430 & -0.488614 \\
\hline SCF Done: & $\begin{array}{l}\mathrm{E}(\mathrm{RM} 062 \mathrm{X}) \\
\mathrm{NFOCk}=9\end{array}$ & $\begin{array}{r}-270.8 \\
o n v=0.6\end{array}$ & $\begin{array}{r}\mathrm{A} . \mathrm{T} \\
-\mathrm{V} / \mathrm{T}=\end{array}$ & $\begin{array}{l}\text { after } \\
.0059\end{array}$ & cycles \\
\hline
\end{tabular}


(b) Thermodynamic quantities at STP

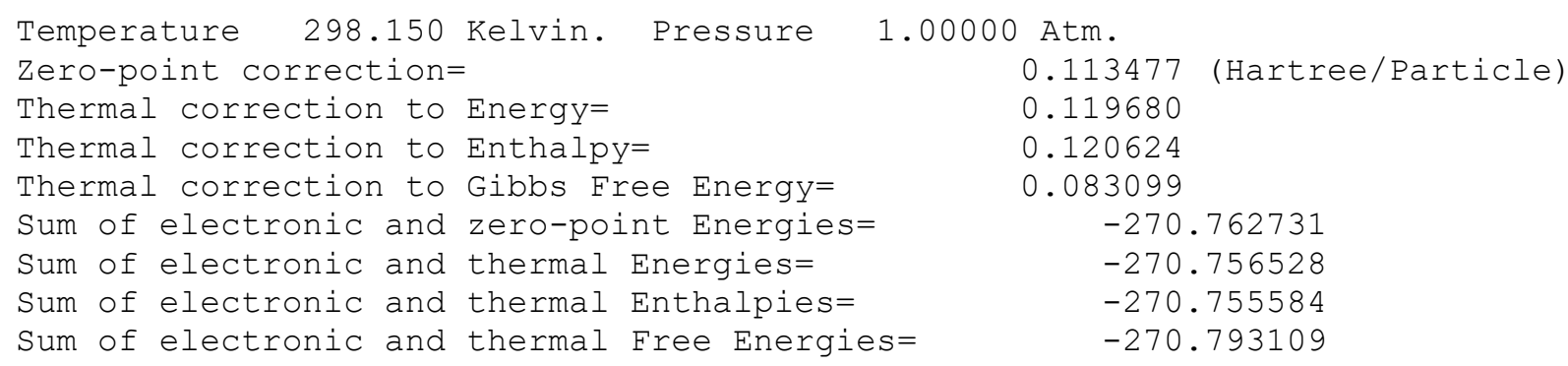

(c) aug-cc-pVTZ single point energy

$$
\begin{array}{llrl}
\mathrm{SCF} \text { Done: } & \mathrm{E}(\mathrm{RM} 062 \mathrm{X})=-270.884849754 & \text { A.U. after } & 13 \text { cycles } \\
\mathrm{NFOCk}=13 & \mathrm{CONV}=0.44 \mathrm{D}-08 & -\mathrm{V} / \mathrm{T}=2.0056 &
\end{array}
$$

(d) Energy in the aqueous phase at STP (SMD model)

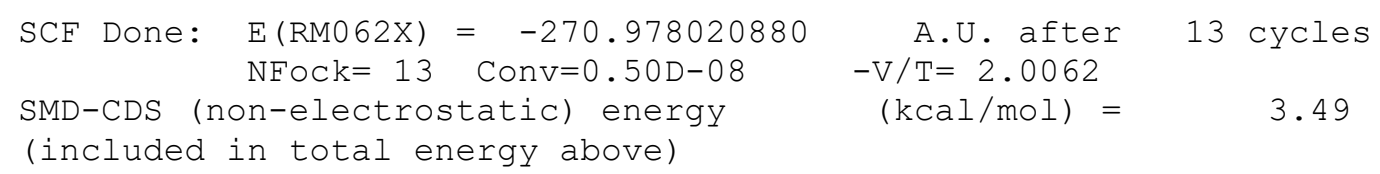

(e) Thermodynamic quantities at non-STP

$\begin{array}{lcc}\text { Temperature } 679.500 \text { Kelvin. } & \text { Pressure } 310.00000 \text { Atm. } & \\ \text { Zero-point correction= } & 0.113477 & \text { (Hartree/Particle) } \\ \text { Thermal correction to Energy= } & 0.141632 & 0.143784 \\ \text { Thermal correction to Enthalpy= } & 0.038095 \\ \text { Thermal correction to Gibbs Free Energy= } & -270.762731 \\ \text { Sum of electronic and zero-point Energies= } & -270.734576 \\ \text { Sum of electronic and thermal Energies= } & -270.732424 \\ \text { Sum of electronic and thermal Enthalpies= } & -270.838113 \\ \text { Sum of electronic and thermal Free Energies= } & \end{array}$

(f) Energy in the aqueous phase at non-STP (SMD model)

$\begin{array}{cccc}\mathrm{SCF} \text { Done: } \quad \mathrm{E}(\mathrm{RM} 062 \mathrm{X})=-270.954236406 & \mathrm{~A} . \mathrm{U} \text {. after } & 14 \text { cycles } \\ \mathrm{NFOCk}=14 \text { Conv=0.75D-08 } & -\mathrm{V} / \mathrm{T}=2.0062 & \\ \text { SMD-CDS (non-electrostatic) energy } & (\mathrm{kcal} / \mathrm{mol})= & 3.49 \\ \text { (included in total energy above) } & \end{array}$

\section{Table S9. n-Butylbenzene (neutral)}

\begin{tabular}{|c|c|c|c|c|c|}
\hline \multirow{2}{*}{$\begin{array}{l}\text { Center } \\
\text { Number }\end{array}$} & \multirow{2}{*}{$\begin{array}{l}\text { Atomic } \\
\text { Number }\end{array}$} & \multirow{2}{*}{$\begin{array}{c}\text { Atomic } \\
\text { Type }\end{array}$} & \multicolumn{3}{|c|}{ Coordinates (Angstroms) } \\
\hline & & & $\mathrm{x}$ & Y & Z \\
\hline 1 & 6 & 0 & 2.642956 & 1.199032 & -0.278764 \\
\hline 2 & 6 & 0 & 1.351268 & 1.195682 & 0.227220 \\
\hline 3 & 6 & 0 & 0.687377 & -0.000000 & 0.487331 \\
\hline 4 & 6 & 0 & 1.351268 & -1.195682 & 0.227220 \\
\hline 5 & 6 & 0 & 2.642956 & -1.199032 & -0.278764 \\
\hline 6 & 6 & 0 & 3.293385 & 0.000000 & -0.533822 \\
\hline 7 & 1 & 0 & 3.143807 & 2.138527 & -0.470869 \\
\hline 8 & 1 & 0 & 0.848428 & 2.134463 & 0.428370 \\
\hline 9 & 1 & 0 & 0.848428 & -2.134463 & 0.428370 \\
\hline
\end{tabular}

(a) Geometry 


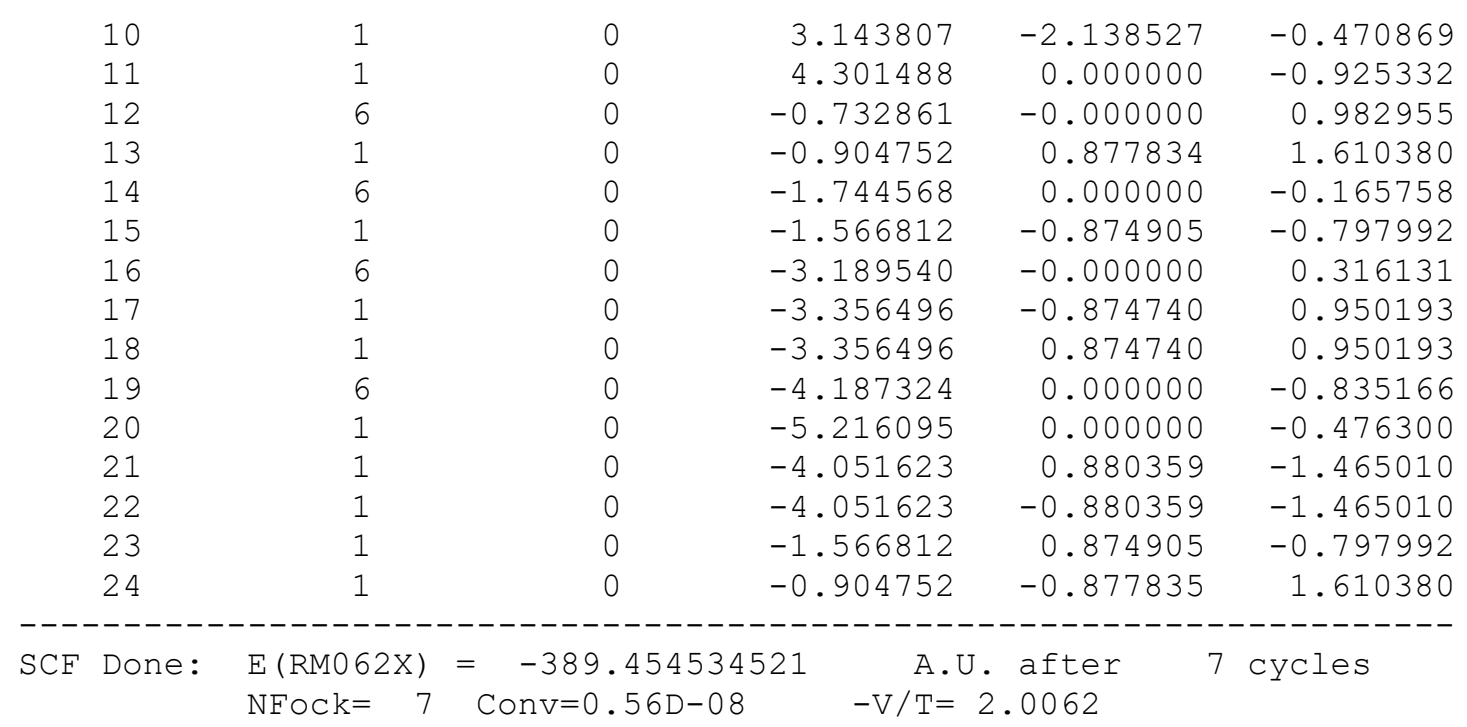

(b) Thermodynamic quantities at STP

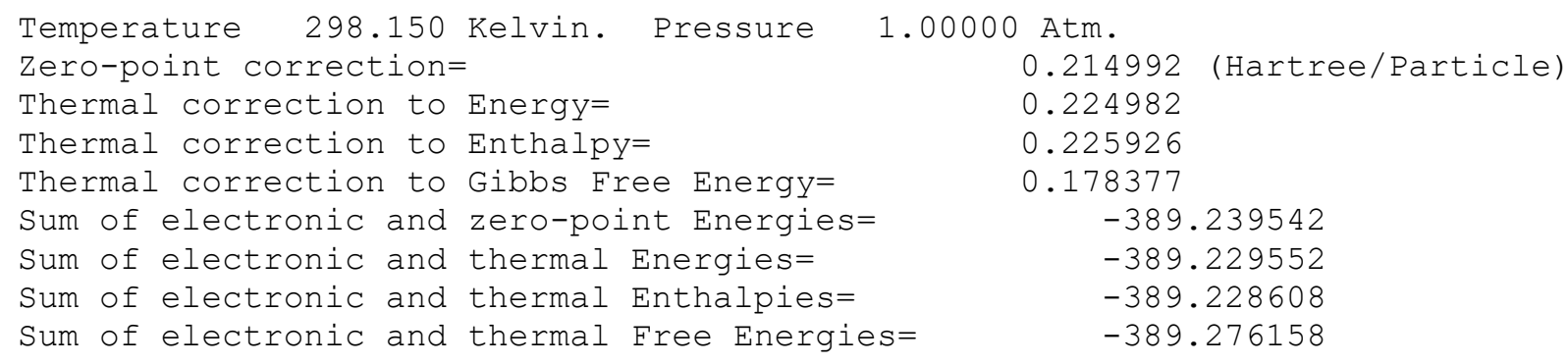

(c) aug-cc-pVTZ single point energy

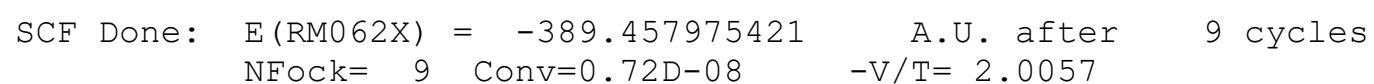

(d) Energy in the aqueous phase at STP (SMD model)

$\begin{array}{cccc}\mathrm{SCF} \text { Done: } & \mathrm{E}(\mathrm{RM} 062 \mathrm{X})=-389.455148642 & \mathrm{~A} . \mathrm{U} . \text { after } & 11 \text { cycles } \\ \mathrm{NFOCk}=11 \text { Conv=0.41D-08 } & -\mathrm{V} / \mathrm{T}=2.0061 & \\ \text { SMD-CDS (non-electrostatic) energy } & (\mathrm{kcal} / \mathrm{mol})= & 4.10 \\ \text { (included in total energy above) } & & \end{array}$

(e) Thermodynamic quantities at non-STP

$\begin{array}{lcc}\text { Temperature } 679.500 \text { Kelvin. } & \text { Pressure } 310.00000 \text { Atm. } \\ \text { Zero-point correction= } & 0.214992 & \text { (Hartree/Particle) } \\ \text { Thermal correction to Energy= } & 0.261520 \\ \text { Thermal correction to Enthalpy= } & 0.263671 \\ \text { Thermal correction to Gibbs Free Energy= } & 0.114821 \\ \text { Sum of electronic and zero-point Energies= } & -389.239542 \\ \text { Sum of electronic and thermal Energies= } & -389.193015 \\ \text { Sum of electronic and thermal Enthalpies= } & -389.190863 \\ \text { Sum of electronic and thermal Free Energies= } & -389.339714\end{array}$

(f) Energy in the aqueous phase at non-STP (SMD model)

$\begin{array}{cccc}\mathrm{SCF} \text { Done: } \mathrm{E}(\mathrm{RM} 062 \mathrm{X})=-389.452943762 & \mathrm{~A} . \mathrm{U} \text {. after } & 10 \text { cycles } \\ \mathrm{NFOCk}=10 \quad \text { Conv=0.89D-08 } & -\mathrm{V} / \mathrm{T}=2.0061 & \\ \text { SMD-CDS (non-electrostatic) energy } & (\mathrm{kcal} / \mathrm{mol})= & 4.10 \\ \text { (included in total energy above) } & \end{array}$


Table S10. n-Butylbenzene: $\alpha$-anion

(a) Geometry

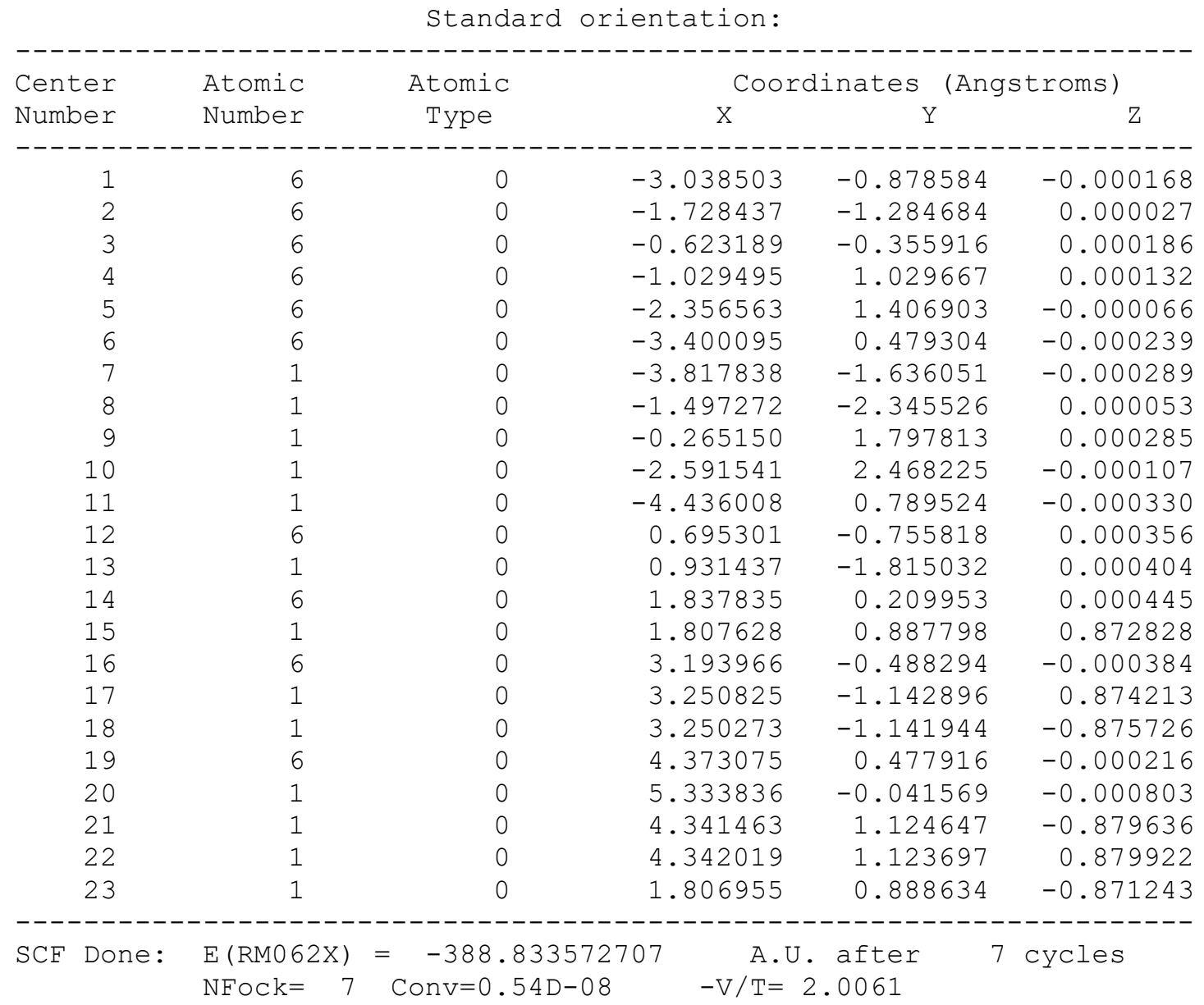

(b) Thermodynamic quantities at STP

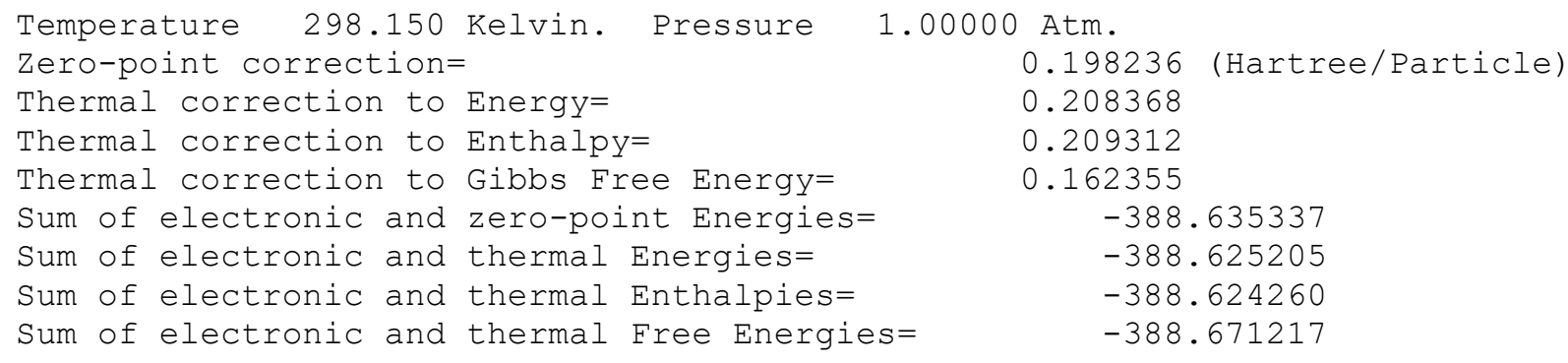

(c) aug-cc-pVTZ single point energy

$\begin{array}{llrl}\mathrm{SCF} \text { Done: } & \mathrm{E}(\mathrm{RM} 062 \mathrm{X})=-388.841443104 & \text { A.U. after } & 12 \text { cycles } \\ & \text { NFock=12 Conv=0.95D-08 } & -\mathrm{V} / \mathrm{T}=2.0056\end{array}$

(d) Energy in the aqueous phase at STP (SMD model)

$\begin{array}{cccc}\mathrm{SCF} \text { Done: } & \mathrm{E}(\mathrm{RM} 062 \mathrm{X})=-388.913278146 & \mathrm{~A} . \mathrm{U} . \text { after } & 13 \text { cycles } \\ \mathrm{NFock}=13 \quad \text { Conv=0.61D-08 } & -\mathrm{V} / \mathrm{T}=2.0060 & \\ \text { SMD-CDS (non-electrostatic) energy } & (\mathrm{kcal} / \mathrm{mol})= & 4.19 \\ \text { (included in total energy above) } & \end{array}$


(e) Thermodynamic quantities at non-STP

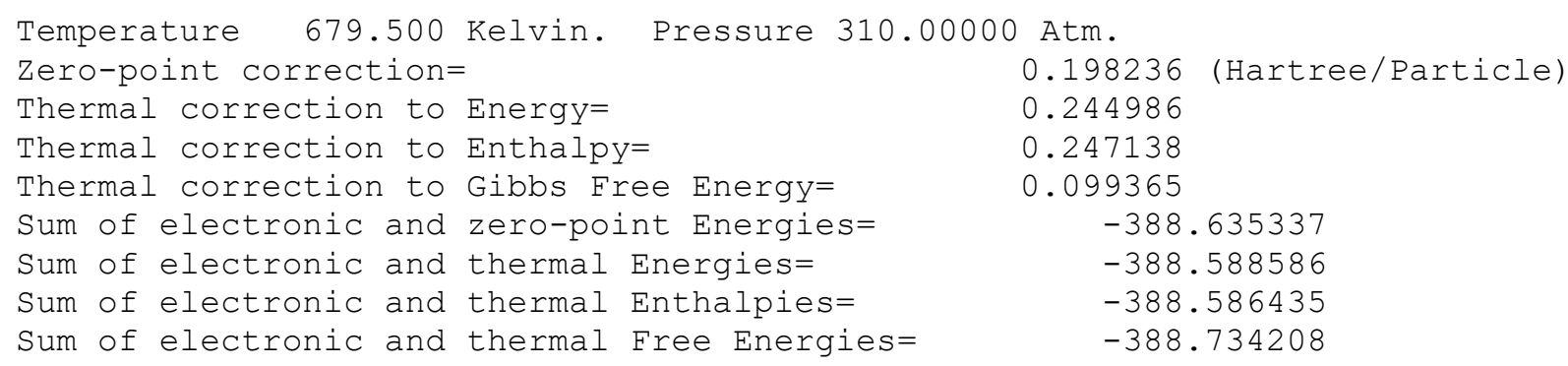

(f) Energy in the aqueous phase at non-STP (SMD model)

$\begin{array}{cccc}\mathrm{SCF} \text { Done: } \mathrm{E}(\mathrm{RM} 062 \mathrm{X})=-388.894527880 & \mathrm{~A} . \mathrm{U} \text {. after } & 13 \text { cycles } \\ \mathrm{NFOCk}=13 \quad \text { Conv=0.48D-08 } & -\mathrm{V} / \mathrm{T}=2.0060 & \\ \text { SMD-CDS (non-electrostatic) energy } & (\mathrm{kcal} / \mathrm{mol})= & 4.19 \\ \text { (included in total energy above) } & & \end{array}$

\section{Table S11. n-Butylbenzene: $\beta$-anion}

(a) Geometry

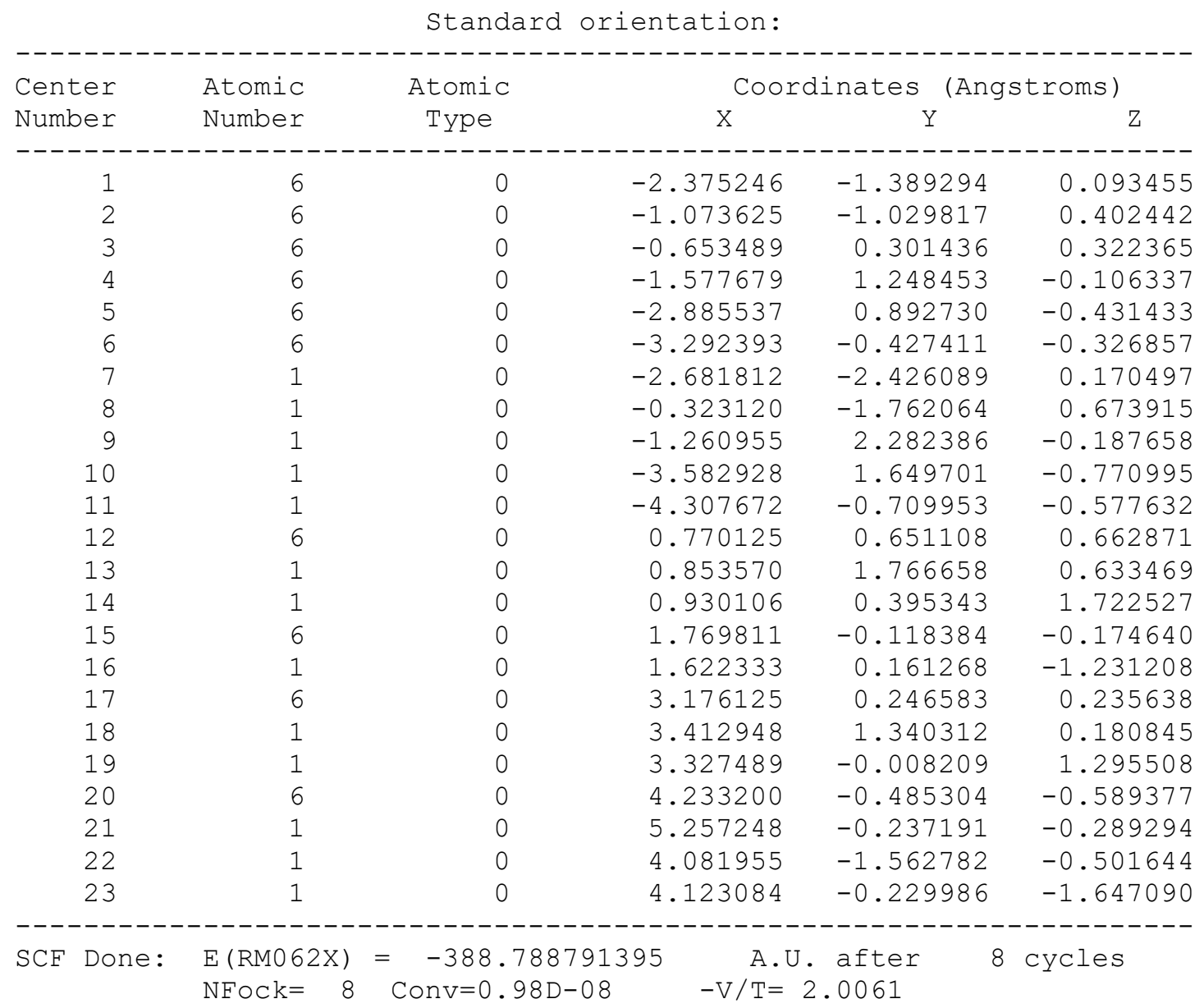

(b) Thermodynamic quantities at STP
Temperature 298.150 Kelvin. Pressure
1.00000 Atm.
Zero-point correction=
0.197354 (Hartree/Particle)
Thermal correction to Energy=
0.207286 
Thermal correction to Enthalpy=

Thermal correction to Gibbs Free Energy=

Sum of electronic and zero-point Energies=

Sum of electronic and thermal Energies=

Sum of electronic and thermal Enthalpies=

Sum of electronic and thermal Free Energies=

$$
\begin{aligned}
0.208231 \\
0.161136 \\
-388.591438 \\
-388.581505 \\
-388.580561 \\
-388.627656
\end{aligned}
$$

(c) aug-cc-pVTZ single point energy

$$
\begin{array}{llrr}
\mathrm{SCF} \text { Done: } & \mathrm{E}(\mathrm{RM} 062 \mathrm{X})=-388.797755484 & \mathrm{~A} . \mathrm{U} \text {. after } & 16 \text { cycles } \\
\mathrm{NFOCk}=16 & \mathrm{COnV}=0.47 \mathrm{D}-08 & -\mathrm{V} / \mathrm{T}=2.0057
\end{array}
$$

(d) Energy in the aqueous phase at STP (SMD model)

$\begin{array}{cccc}\mathrm{SCF} \text { Done: } \mathrm{E}(\mathrm{RM} 062 \mathrm{X})=-388.876757656 & \mathrm{~A} . \mathrm{U} \text {. after } & 13 \text { cycles } \\ \mathrm{NFock}=13 \quad \text { Conv=0.76D-08 } & -\mathrm{V} / \mathrm{T}=2.0061 & \\ \text { SMD-CDS (non-electrostatic) energy } & (\mathrm{kcal} / \mathrm{mol})= & 4.28 \\ \text { (included in total energy above) } & \end{array}$

\begin{tabular}{|c|c|c|c|c|c|}
\hline \multirow{2}{*}{$\begin{array}{l}\text { Center } \\
\text { Number }\end{array}$} & \multirow{2}{*}{$\begin{array}{l}\text { Atomic } \\
\text { Number }\end{array}$} & \multirow{2}{*}{$\begin{array}{c}\text { Atomic } \\
\text { Type }\end{array}$} & \multicolumn{3}{|c|}{ Coordinates (Angstroms) } \\
\hline & & & $\mathrm{X}$ & Y & Z \\
\hline & & & 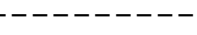 & --- & --------1 \\
\hline 1 & 6 & 0 & -2.531229 & -1.262446 & -0.188307 \\
\hline 2 & 6 & 0 & -1.242170 & -1.154319 & 0.313948 \\
\hline 3 & 6 & 0 & -0.632097 & 0.088974 & 0.492144 \\
\hline 4 & 6 & 0 & -1.366167 & 1.221945 & 0.141772 \\
\hline 5 & 6 & 0 & -2.656923 & 1.123253 & -0.360694 \\
\hline 6 & 6 & 0 & -3.248043 & -0.121852 & -0.527555 \\
\hline 7 & 1 & 0 & -2.978946 & -2.240194 & -0.317885 \\
\hline 8 & 1 & 0 & -0.683438 & -2.045757 & 0.572834 \\
\hline 9 & 1 & 0 & -0.904517 & 2.194720 & 0.263570 \\
\hline 10 & 1 & 0 & -3.202974 & 2.020951 & -0.623765 \\
\hline 11 & 1 & 0 & -4.254154 & -0.203170 & -0.918586 \\
\hline 12 & 6 & 0 & 0.782032 & 0.198274 & 0.976774 \\
\hline 13 & 1 & 0 & 0.971118 & -0.558375 & 1.744197 \\
\hline 14 & 6 & 0 & 1.822560 & 0.011536 & -0.140817 \\
\hline
\end{tabular}

(e) Thermodynamic quantities at non-STP

$\begin{array}{lcc}\text { Temperature } 679.500 \text { Kelvin. } & \text { Pressure 310.00000 Atm. } \\ \text { Zero-point correction= } & 0.197354 \text { (Hartree/Particle) } \\ \text { Thermal correction to Energy= } & 0.243562 \\ \text { Thermal correction to Enthalpy= } & 0.245714 \\ \text { Thermal correction to Gibbs Free Energy= } & 0.098193 \\ \text { Sum of electronic and zero-point Energies= } & -388.591438 \\ \text { Sum of electronic and thermal Energies= } & -388.545229 \\ \text { Sum of electronic and thermal Enthalpies= } & -388.543078 \\ \text { Sum of electronic and thermal Free Energies= } & -388.690599\end{array}$

(f) Energy in the aqueous phase at non-STP (SMD model)

$\begin{array}{cccc}\mathrm{SCF} \text { Done: } & \mathrm{E}(\mathrm{RM} 062 \mathrm{X})=-388.854562565 & \mathrm{~A} . \mathrm{U} \text {. after } & 13 \text { cycles } \\ \mathrm{NFock}=13 & \text { Conv=0.89D-08 } & -\mathrm{V} / \mathrm{T}=2.0061 & \\ \mathrm{SMD}-\mathrm{CDS} \text { (non-electrostatic) energy } & (\mathrm{kcal} / \mathrm{mol})= & 4.28\end{array}$

(included in total energy above)

\section{Table S12. n-Butylbenzene: $\gamma$-anion}

\section{(a) Geometry}




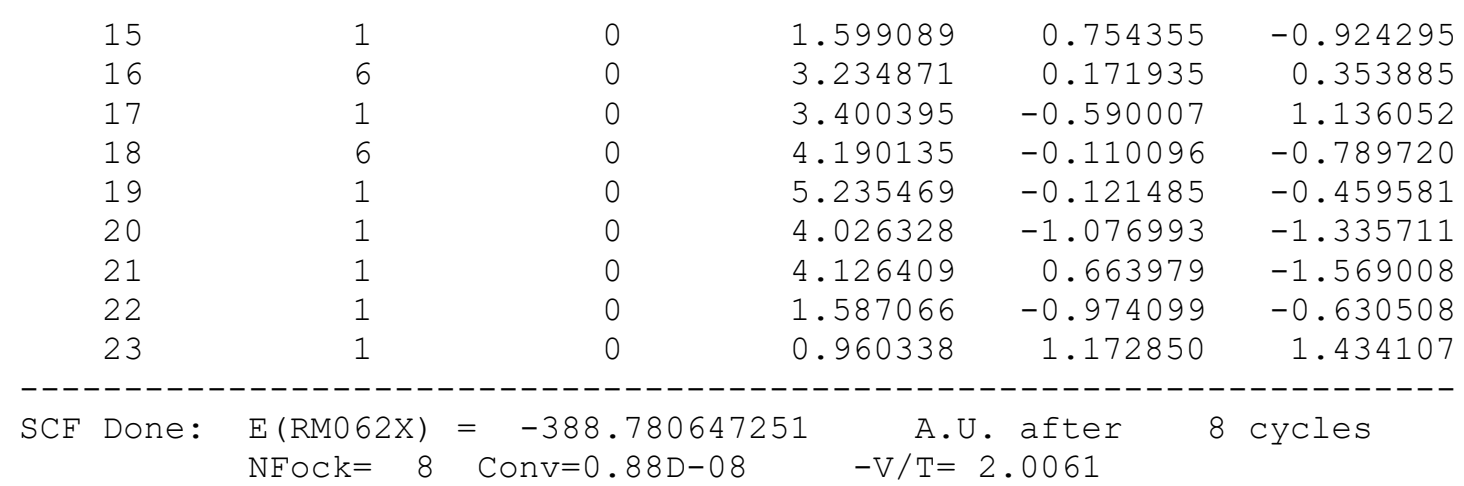

(b) Thermodynamic quantities at STP

$\begin{array}{lcc}\text { Temperature } 298.150 \text { Kelvin. } & \text { Pressure } & 1.00000 \text { Atm. } \\ \text { Zero-point correction= } & 0.197047 & \text { (Hartree/Particle) } \\ \text { Thermal correction to Energy= } & 0.207037 \\ \text { Thermal correction to Enthalpy= } & 0.207981 \\ \text { Thermal correction to Gibbs Free Energy= } & 0.160445 \\ \text { Sum of electronic and zero-point Energies= } & -388.583600 \\ \text { Sum of electronic and thermal Energies= } & -388.573610 \\ \text { Sum of electronic and thermal Enthalpies= } & -388.572666 \\ \text { Sum of electronic and thermal Free Energies= } & -388.620202\end{array}$

(c) aug-cc-pVTZ single point energy

$$
\begin{array}{llrl}
\mathrm{SCF} \text { Done: } & \mathrm{E}(\mathrm{RM} 062 \mathrm{X})=-388.791618587 & \mathrm{~A} . \mathrm{U} \text {. after } & 18 \text { cycles } \\
\text { NFock }=18 & \text { Conv=0.19D-08 } & -\mathrm{V} / \mathrm{T}=2.0057
\end{array}
$$

(d) Energy in the aqueous phase at STP (SMD model)

$\begin{array}{cccc}\mathrm{SCF} \text { Done: } \mathrm{E}(\mathrm{RM} 062 \mathrm{X})=-388.875659888 & \mathrm{~A} . \mathrm{U} \text {. after } & 13 \text { cycles } \\ \mathrm{NFOCk}=13 \quad \text { Conv=0.53D-08 } & -\mathrm{V} / \mathrm{T}=2.0061 & \\ \text { SMD-CDS (non-electrostatic) energy } & (\mathrm{kcal} / \mathrm{mol})= & 4.31 \\ \text { (included in total energy above) } & \end{array}$

(e) Thermodynamic quantities at non-STP

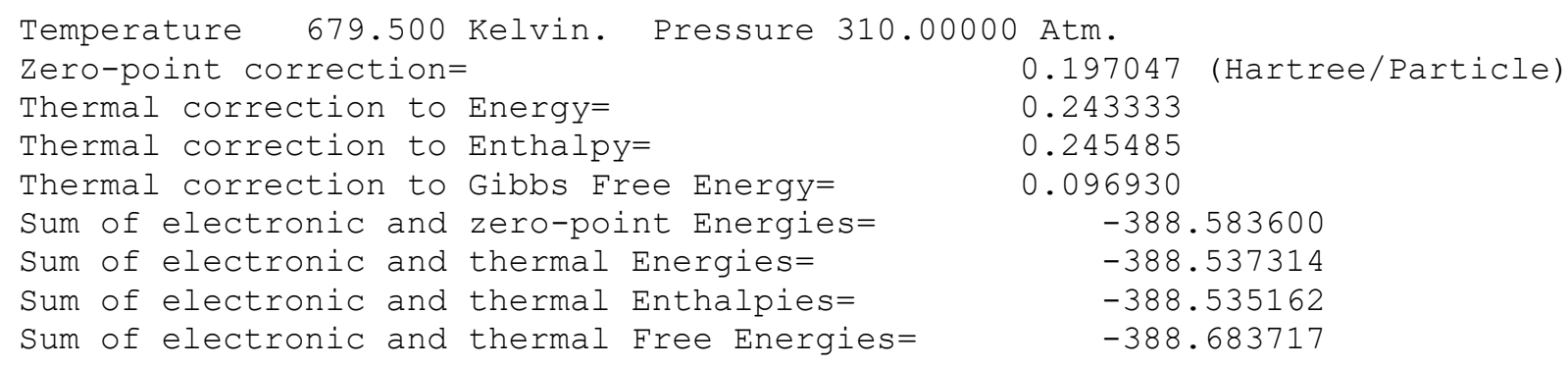

(f) Energy in the aqueous phase at non-STP (SMD model)

$\begin{array}{cccc}\mathrm{SCF} \text { Done: } & \mathrm{E}(\mathrm{RM} 062 \mathrm{X})=-388.852588155 & \mathrm{~A} . \mathrm{U} \text {. after } & 13 \text { cycles } \\ \mathrm{NFOCk}=13 \text { Conv=0.50D-08 } & -\mathrm{V} / \mathrm{T}=2.0061 & \\ \text { SMD-CDS (non-electrostatic) energy } & (\mathrm{kcal} / \mathrm{mol})= & 4.31 \\ \text { (included in total energy above) } & \end{array}$

\section{Table S13. n-Butylbenzene: $\delta$-anion}

(a) Geometry

Standard orientation: 


\begin{tabular}{|c|c|c|c|c|c|}
\hline \multirow{2}{*}{$\begin{array}{l}\text { Center } \\
\text { Number }\end{array}$} & \multirow{2}{*}{$\begin{array}{l}\text { Atomic } \\
\text { Number }\end{array}$} & \multirow{2}{*}{$\begin{array}{c}\text { Atomic } \\
\text { Type }\end{array}$} & \multicolumn{3}{|c|}{ Coordinates (Angstroms) } \\
\hline & & & $\mathrm{X}$ & $\mathrm{Y}$ & $\mathrm{Z}$ \\
\hline 1 & 6 & 0 & 2.602274 & 1.197639 & -0.262571 \\
\hline 2 & 6 & 0 & 1.303415 & 1.194340 & 0.221647 \\
\hline 3 & 6 & 0 & 0.624313 & 0.000000 & 0.476753 \\
\hline 4 & 6 & 0 & 1.303415 & -1.194340 & 0.221647 \\
\hline 5 & 6 & 0 & 2.602274 & -1.197639 & -0.262571 \\
\hline 6 & 6 & 0 & 3.263203 & 0.000000 & -0.508392 \\
\hline 7 & 1 & 0 & 3.103245 & 2.139461 & -0.449497 \\
\hline 8 & 1 & 0 & 0.792611 & 2.132345 & 0.405690 \\
\hline 9 & 1 & 0 & 0.792611 & -2.132345 & 0.405690 \\
\hline 10 & 1 & 0 & 3.103245 & -2.139461 & -0.449497 \\
\hline 11 & 1 & 0 & 4.276864 & 0.000000 & -0.886849 \\
\hline 12 & 6 & 0 & -0.796344 & 0.000000 & 0.941450 \\
\hline 13 & 1 & 0 & -0.984908 & 0.879414 & 1.564075 \\
\hline 14 & 6 & 0 & -1.814361 & 0.000000 & -0.217594 \\
\hline 15 & 1 & 0 & -1.646035 & -0.876961 & -0.850621 \\
\hline 16 & 6 & 0 & -3.290183 & 0.000000 & 0.262190 \\
\hline 17 & 1 & 0 & -3.389549 & 0.874566 & 0.927371 \\
\hline 18 & 6 & 0 & -4.380713 & 0.000000 & -0.781013 \\
\hline 19 & 1 & 0 & -4.265677 & 0.886167 & -1.428235 \\
\hline 20 & 1 & 0 & -4.265677 & -0.886167 & -1.428235 \\
\hline 21 & 1 & 0 & -1.646035 & 0.876961 & -0.850621 \\
\hline 22 & 1 & 0 & -0.984908 & -0.879414 & 1.564075 \\
\hline 23 & 1 & 0 & -3.389549 & -0.874566 & 0.927371 \\
\hline Don & $\begin{array}{l}\text { E (RM0 62X) } \\
\text { NFock } \quad 9\end{array}$ & $\begin{array}{r}-3 \\
\text { Conv }\end{array}$ & $-V / T=$ & $\begin{array}{l}\text { after } \\
.0060\end{array}$ & cycles \\
\hline
\end{tabular}

(b) Thermodynamic quantities at STP

$\begin{array}{lcc}\text { Temperature } 298.150 \text { Kelvin. } & \text { Pressure } & 1.00000 \text { Atm. } \\ \text { Zero-point correction= } & 0.197497 & \text { (Hartree/Particle) } \\ \text { Thermal correction to Energy= } & 0.207471 & 0.208416 \\ \text { Thermal correction to Enthalpy= } & 0.161218 \\ \text { Thermal correction to Gibbs Free Energy= } & -388.579553 \\ \text { Sum of electronic and zero-point Energies= } & -388.569578 \\ \text { Sum of electronic and thermal Energies= } & -388.568634 \\ \text { Sum of electronic and thermal Enthalpies= } & -388.615832 \\ \text { Sum of electronic and thermal Free Energies= }\end{array}$

(c) aug-cc-pVTZ single point energy

$\begin{array}{llrl}\mathrm{SCF} \text { Done: } & \mathrm{E}(\mathrm{RM} 062 \mathrm{X})=-388.789647623 & \text { A.U. after } & 18 \text { cycles } \\ & \mathrm{NFOCk}=18 \quad \mathrm{Conv}=0.31 \mathrm{D}-08 & -\mathrm{V} / \mathrm{T}=2.0057\end{array}$

(d) Energy in the aqueous phase at STP (SMD model)

$\begin{array}{cccc}\mathrm{SCF} \text { Done: } \quad \mathrm{E}(\mathrm{RM} 062 \mathrm{X})=-388.880389729 & \begin{array}{c}\mathrm{A} . \mathrm{U} . \text { after } \\ \text { NFock=13 Conv=0.53D-08 }\end{array} & -\mathrm{V} / \mathrm{T}=2.0062 \\ \text { SMD-CDS (non-electrostatic) energy } & (\mathrm{kcal} / \mathrm{mol})= & 4.81 \\ \text { (included in total energy above) } & \end{array}$

(e) Thermodynamic quantities at non-STP

$\begin{array}{lrr}\text { Temperature } 679.500 \text { Kelvin. } & \text { Pressure } 310.00000 \text { Atm. } & \\ \text { Zero-point correction= } & 0.197497 & \text { (Hartree/Particle) } \\ \text { Thermal correction to Energy= } & 0.244030\end{array}$


Thermal correction to Enthalpy=

Thermal correction to Gibbs Free Energy=

Sum of electronic and zero-point Energies=

Sum of electronic and thermal Energies=

Sum of electronic and thermal Enthalpies=

Sum of electronic and thermal Free Energies=
0.246182

0.098000

$-388.579553$

$-388.533019$

$-388.530867$

$-388.679049$

(f) Energy in the aqueous phase at non-STP (SMD model)

$\begin{array}{ccccc}\mathrm{SCF} \text { Done: } & \mathrm{E}(\mathrm{RM} 062 \mathrm{X})=-388.855364128 & \mathrm{~A} . \mathrm{U} \text {. after } & 14 \text { cycles } \\ \mathrm{NFock}=14 \quad \text { Conv=0.13D-08 } & -\mathrm{V} / \mathrm{T}=2.0062 & \\ \mathrm{SMD}-\mathrm{CDS} \text { (non-electrostatic) energy } & (\mathrm{kcal} / \mathrm{mol})= & 4.81\end{array}$

(included in total energy above)

\section{Table S14. n-Butylbenzene: ortho-anion}

\section{(a) Geometry}

Standard orientation:

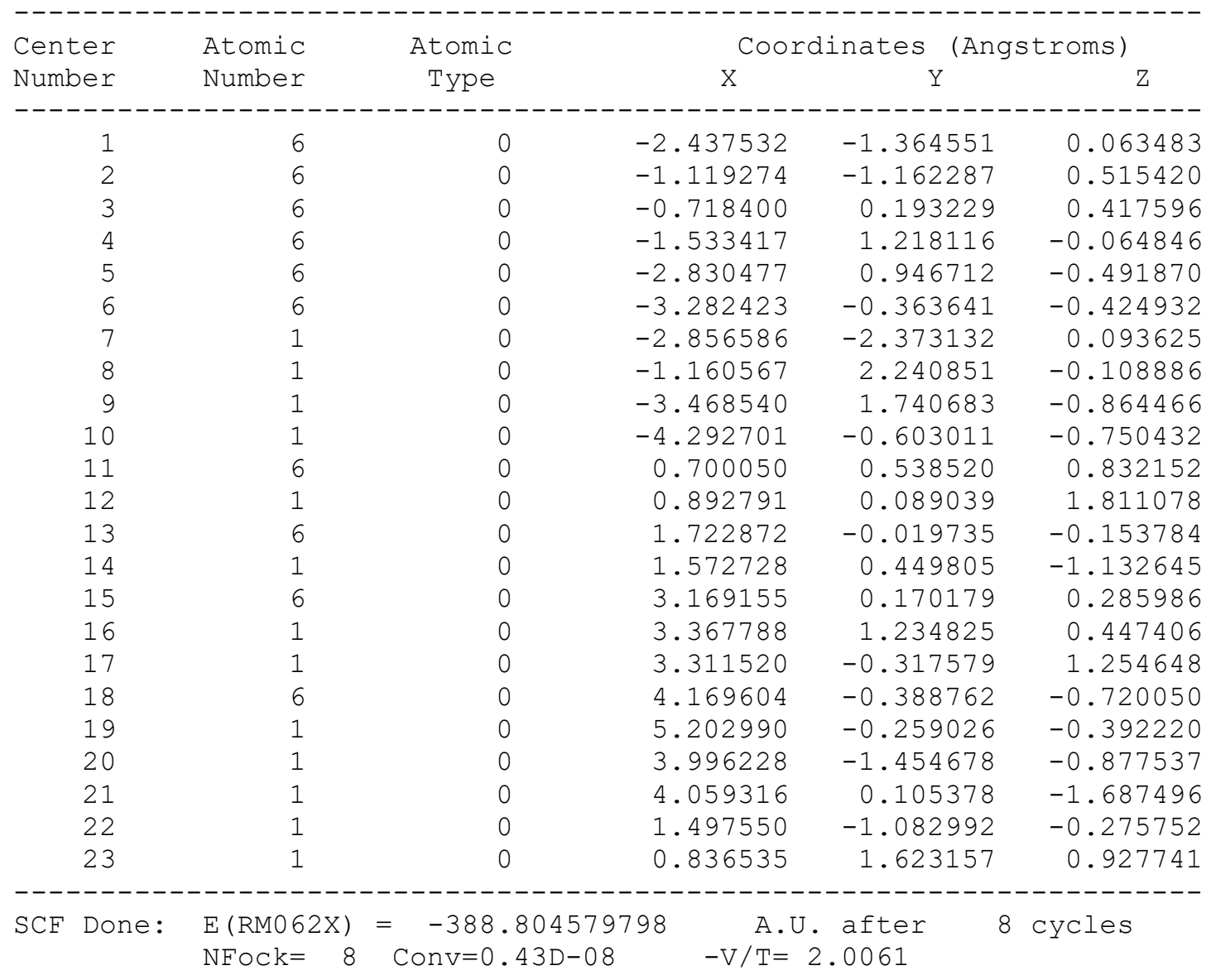

(b) Thermodynamic quantities at STP

$\begin{array}{lcc}\text { Temperature } 298.150 \text { Kelvin. } & \text { Pressure } & 1.00000 \text { Atm. } \\ \text { Zero-point correction= } & 0.199647 & \text { (Hartree/Particle) } \\ \text { Thermal correction to Energy= } & 0.209597 & \\ \text { Thermal correction to Enthalpy= } & 0.210542 \\ \text { Thermal correction to Gibbs Free Energy= } & 0.163509 \\ \text { Sum of electronic and zero-point Energies= } & -388.604933 \\ \text { Sum of electronic and thermal Energies= } & -388.594982\end{array}$


Sum of electronic and thermal Enthalpies= $\quad-388.594038$

Sum of electronic and thermal Free Energies= $\quad-388.641070$

(c) aug-cc-pVTZ single point energy

$\begin{array}{llrl}\mathrm{SCF} \text { Done: } & \mathrm{E}(\mathrm{RM} 062 \mathrm{X})=-388.812962014 & \mathrm{~A} . \mathrm{U} \text {. after } & 13 \text { cycles } \\ \mathrm{NFOCk}=13 & \mathrm{Conv}=0.10 \mathrm{D}-07 & -\mathrm{V} / \mathrm{T}=2.0057\end{array}$

(d) Energy in the aqueous phase at STP (SMD model)

$\begin{array}{cccc}\mathrm{SCF} \text { Done: } & \mathrm{E}(\mathrm{RM} 6 \mathrm{X})=-388.898699762 & \mathrm{~A} . \mathrm{U} \text {. after } & 13 \text { cycles } \\ \text { NFock=13 Conv=0.61D-08 } & -\mathrm{V} / \mathrm{T}=2.0062 & \\ \text { SMD-CDS (non-electrostatic) energy } & (\mathrm{kcal} / \mathrm{mol})= & 4.33 \\ \text { (included in total energy above) } & & \end{array}$

(e) Thermodynamic quantities at non-STP

$\begin{array}{lcc}\text { Temperature } 679.500 \text { Kelvin. } & \text { Pressure 310.00000 Atm. } \\ \text { Zero-point correction= } & 0.199647 & \text { (Hartree/Particle) } \\ \text { Thermal correction to Energy= } & 0.245541 \\ \text { Thermal correction to Enthalpy= } & 0.247693 \\ \text { Thermal correction to Gibbs Free Energy= } & 0.100783 \\ \text { Sum of electronic and zero-point Energies= } & -388.604933 \\ \text { Sum of electronic and thermal Energies= } & -388.559038 \\ \text { Sum of electronic and thermal Enthalpies= } & -388.556887 \\ \text { Sum of electronic and thermal Free Energies= } & -388.703796\end{array}$

(f) Energy in the aqueous phase at non-STP (SMD model)

$\begin{array}{cccc}\mathrm{SCF} \text { Done: } \mathrm{E}(\mathrm{RM} 062 \mathrm{X})=-388.875517349 & \mathrm{~A} . \mathrm{U} \text {. after } & 13 \text { cycles } \\ \mathrm{NFock}=13 \quad \text { Conv=0.61D-08 } & -\mathrm{V} / \mathrm{T}=2.0062 & \\ \text { SMD-CDS (non-electrostatic) energy } & (\mathrm{kcal} / \mathrm{mol})= & 4.33 \\ \text { (included in total energy above) } & \end{array}$

\section{Table S15. n-Butylbenzene: meta-anion}

(a) Geometry

\begin{tabular}{|c|c|c|c|c|c|}
\hline \multirow{2}{*}{$\begin{array}{l}\text { Center } \\
\text { Number }\end{array}$} & \multirow{2}{*}{$\begin{array}{l}\text { Atomic } \\
\text { Number }\end{array}$} & \multirow{2}{*}{$\begin{array}{c}\text { Atomic } \\
\text { Type }\end{array}$} & \multicolumn{3}{|c|}{ Coordinates (Angstroms) } \\
\hline & & & $\mathrm{X}$ & Y & Z \\
\hline 1 & 6 & 0 & 2.687117 & 1.375824 & -0.294188 \\
\hline 2 & 6 & 0 & 1.385870 & 1.211524 & 0.229941 \\
\hline 3 & 6 & 0 & 0.741788 & -0.005731 & 0.480215 \\
\hline 4 & 6 & 0 & 1.417834 & -1.191751 & 0.207485 \\
\hline 5 & 6 & 0 & 2.707431 & -1.118690 & -0.308624 \\
\hline 6 & 6 & 0 & 3.296391 & 0.122658 & -0.546160 \\
\hline 7 & 1 & 0 & 0.800640 & 2.104436 & 0.479630 \\
\hline 8 & 1 & 0 & 0.943670 & -2.150659 & 0.400053 \\
\hline 9 & 1 & 0 & 3.251123 & -2.037683 & -0.520815 \\
\hline 10 & 1 & 0 & 4.309685 & 0.100650 & -0.954688 \\
\hline 11 & 6 & 0 & -0.682064 & -0.039154 & 0.978728 \\
\hline 12 & 1 & 0 & -0.862498 & 0.820487 & 1.632528 \\
\hline 13 & 6 & 0 & -1.702723 & -0.005944 & -0.160110 \\
\hline 14 & 1 & 0 & -1.525291 & -0.862167 & -0.818329 \\
\hline 15 & 6 & 0 & -3.149422 & -0.017535 & 0.318472 \\
\hline 16 & 1 & 0 & -3.319435 & -0.908577 & 0.930352 \\
\hline 17 & 1 & 0 & -3.316078 & 0.840466 & 0.976266 \\
\hline 18 & 6 & 0 & -4.151838 & 0.014779 & -0.829606 \\
\hline
\end{tabular}




\begin{tabular}{|c|c|c|c|c|c|}
\hline 19 & 1 & 0 & -5.183763 & 0.007415 & -0.474678 \\
\hline 20 & 1 & 0 & -4.011098 & 0.910393 & -1.437142 \\
\hline 21 & 1 & 0 & -4.014947 & -0.847895 & -1.484195 \\
\hline 22 & 1 & 0 & -1.519690 & 0.886728 & -0.765096 \\
\hline 23 & 1 & 0 & -0.854620 & -0.939471 & 1.579212 \\
\hline Done: & $\begin{array}{l}E(\mathrm{RM} 062 \mathrm{X}) \\
\mathrm{NFOCk}=8\end{array}$ & $=\quad-388$ & $\begin{array}{r}A \\
-V / T\end{array}$ & $\begin{array}{l}\text { after } \\
.0061\end{array}$ & cycles \\
\hline
\end{tabular}

(b) Thermodynamic quantities at STP

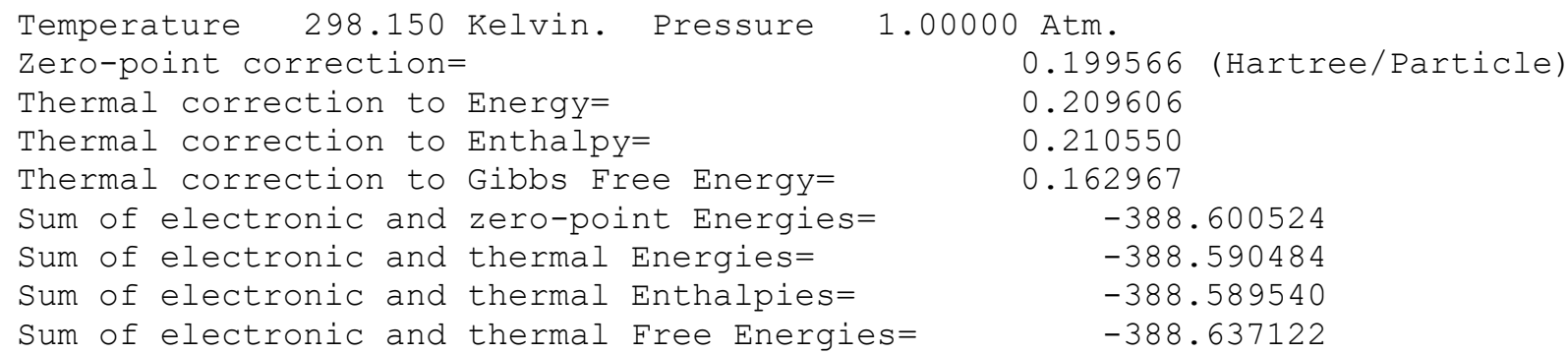

(c) aug-cc-pVTZ single point energy

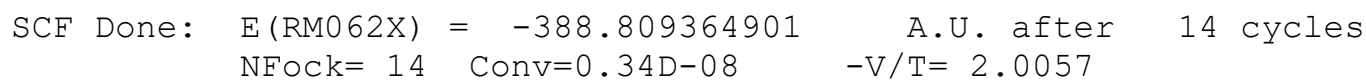

(d) Energy in the aqueous phase at STP (SMD model)

$\begin{array}{ccccc}\mathrm{SCF} \text { Done: } & \mathrm{E}(\mathrm{RM} 062 \mathrm{X})=-388.899141293 & \mathrm{~A} . \mathrm{U} \text {. after } & 13 \text { cycles } \\ \mathrm{NFOCk}=13 & \mathrm{Conv}=0.53 \mathrm{D}-08 & -\mathrm{V} / \mathrm{T}=2.0063 & \\ \text { SMD-CDS (non-electrostatic) energy } & (\mathrm{kcal} / \mathrm{mol})= & 4.38\end{array}$

(e) Thermodynamic quantities at non-STP

$\begin{array}{lcc}\text { Temperature } 679.500 \text { Kelvin. } & \text { Pressure 310.00000 Atm. } \\ \text { Zero-point correction= } & 0.199566 & \text { (Hartree/Particle) } \\ \text { Thermal correction to Energy= } & 0.245512 \\ \text { Thermal correction to Enthalpy= } & 0.247663 \\ \text { Thermal correction to Gibbs Free Energy= } & 0.099551 \\ \text { Sum of electronic and zero-point Energies= } & -388.600524 \\ \text { Sum of electronic and thermal Energies= } & -388.554578 \\ \text { Sum of electronic and thermal Enthalpies= } & -388.552426 \\ \text { Sum of electronic and thermal Eree Energies= } & -388.700539\end{array}$

(f) Energy in the aqueous phase at non-STP (SMD model)

$\begin{array}{ccccc}\mathrm{SCF} \text { Done: } & \mathrm{E}(\mathrm{RM} 062 \mathrm{X})=-388.875210422 & \mathrm{~A} . \mathrm{U} \text {. after } & 14 \text { cycles } \\ \mathrm{NFOCk}=14 \quad \text { Conv=0.53D-08 } & -\mathrm{V} / \mathrm{T}=2.0062 & \\ \mathrm{SMD}-\mathrm{CDS} \text { (non-electrostatic) energy } & (\mathrm{kcal} / \mathrm{mol})= & 4.38\end{array}$

(included in total energy above)

\section{Table S16. n-Butylbenzene: para-anion}

(a) Geometry

Standard orientation:

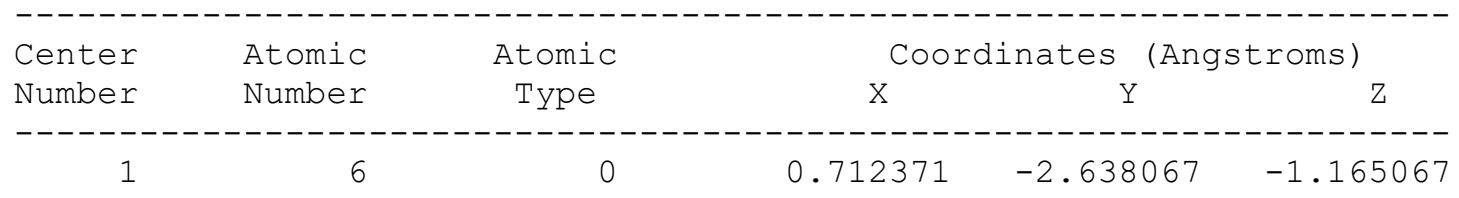




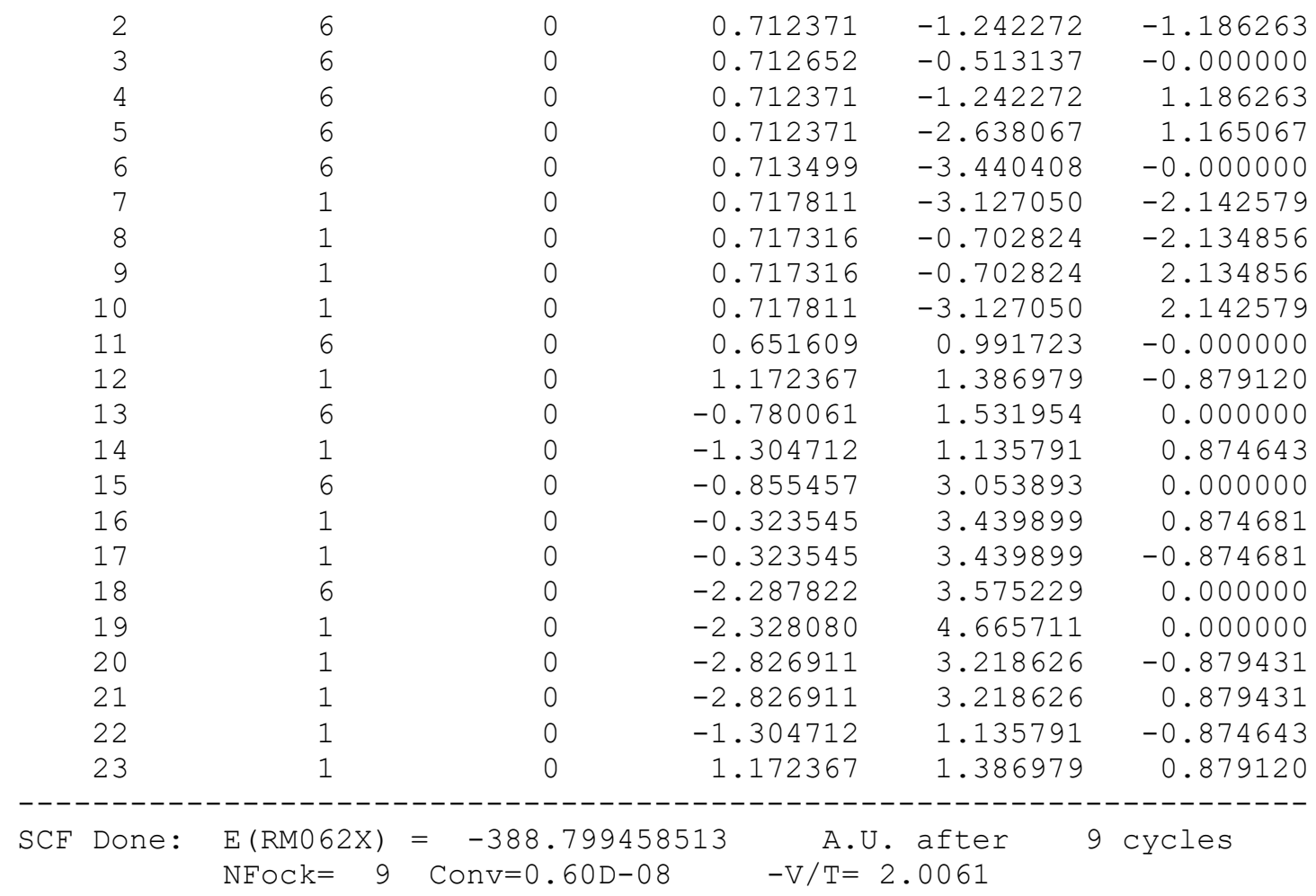

(b) Thermodynamic quantities at STP

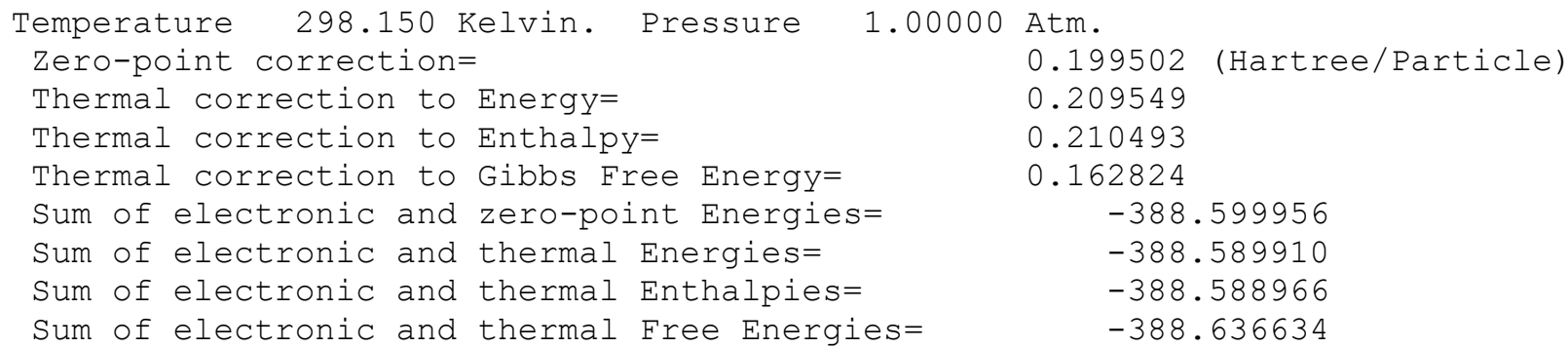

(c) aug-cc-pVTZ single point energy

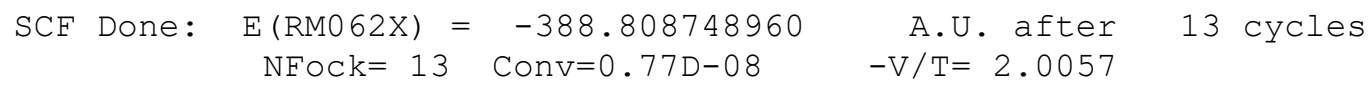

(d) Energy in the aqueous phase at STP (SMD model)

$\begin{array}{cccc}\mathrm{SCF} \text { Done: } \mathrm{E}(\mathrm{RM} 062 \mathrm{X})=-388.898843728 & \begin{array}{c}\mathrm{A} . \mathrm{U} \cdot \text { after } \\ \text { NFock=13 cycles }\end{array} \\ \text { SMD-CDS (non-electrostatic) energy } & \begin{array}{c}\mathrm{V} / \mathrm{T}=2.0063 \\ (\mathrm{kcal} / \mathrm{mol})=\end{array} & 4.38 \\ \text { (included in total energy above) } & \end{array}$

(e) Thermodynamic quantities at non-STP

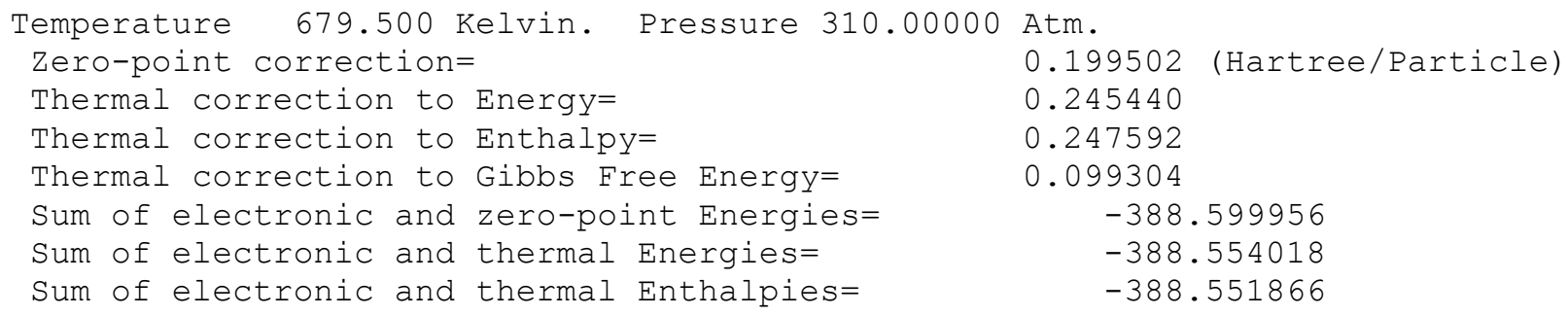


Sum of electronic and thermal Free Energies=

$-388.700155$

(f) Energy in the aqueous phase at non-STP (SMD model)

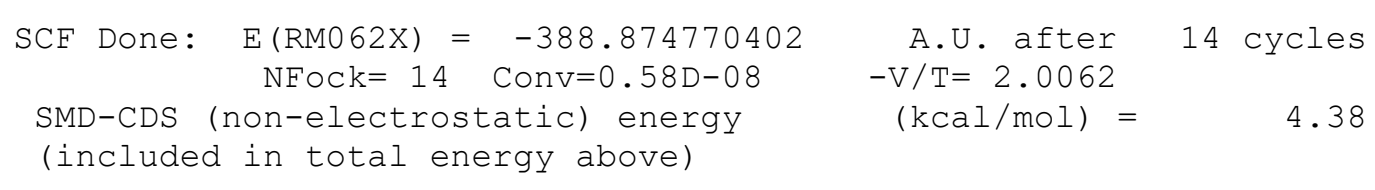




\section{Table S17. Relaxed PE Scans}

(UCAM-B3LYP/cc-pVDZ with SMD solvation at STP)

\begin{tabular}{|c|c|c|c|c|c|c|c|c|c|c|c|c|c|}
\hline \multicolumn{2}{|c|}{$\alpha$} & \multicolumn{2}{|c|}{$\beta$} & \multicolumn{2}{|r|}{$\gamma$} & \multicolumn{2}{|c|}{$\delta$} & \multicolumn{2}{|c|}{ ortho } & \multicolumn{2}{|c|}{ meta } & \multicolumn{2}{|c|}{ para } \\
\hline $\operatorname{RCH}_{\text {CH }}(\AA)$ & $E(a u)$ & $\mathrm{R}_{\mathrm{CH}}(\AA \AA)$ & $E(a u)$ & $\mathrm{CH}(\AA \AA)$ & $E(a u)$ & $\operatorname{R}_{\mathrm{CH}}(\AA \AA)$ & $E(a u)$ & :H $(\AA ̊)$ & $E(a u)$ & $\operatorname{R}_{C H}(\AA)$ & $E(a u)$ & $\operatorname{RCH}_{\text {CH }}(\AA)$ & $E(a u)$ \\
\hline 926 & 9010 & 942 & 65.168971 & 0418 & 164798 & 9012 & -465.15797 & 838 & -465.16941 & .08216 & 60180 & 1.0817 & 61080 \\
\hline 1.1926 & 65.164847 & 1942 & -465.164573 & 20418 & 65.159869 & 19012 & -465.15386 & 1838 & -465.16553 & 18216 & 6980 & 1817 & 880 \\
\hline 1.2926 & 3981 & 2942 & 585 & 30418 & -465.148626 & 29012 & . & 838 & & 28216 & & & \\
\hline 1.3926 & -465.141663 & 1.3942 & -465.140872 & 1.40418 & -465.134943 & 1.39012 & -465.13156 & 1.3838 & -465.14652 & 1.38216 & -465.141350 & 1.3817 & -465 \\
\hline 1.4926 & 79 & 942 & 46 & 8 & 96 & & & & & & & & \\
\hline 1.5926 & -465.142730 & 1.5942 & -465.128040 & 1.60418 & -465.123 & 1.59012 & -465.12416 & 8838 & -465.1 & 1.58216 & -46 & 17 & \\
\hline 1.6926 & 1 & 2 & & & & & & & & & & & \\
\hline 1.7926 & -465.144233 & 1.7942 & -465.124270 & 1.80418 & -465.120522 & 1.79012 & 2175 & 1.7838 & -46 & 1.78216 & & 7 & 17 \\
\hline 1.8926 & -465.144261 & 1.8942 & -465.122656 & 1.90418 & -465 & 1.89012 & -465 & 1.8838 & & 1.88216 & & & \\
\hline 1.9926 & -465.143886 & 9942 & 0974 & 2.00418 & 465 & 1.99012 & 189 & 338 & -46 & 1.9 & & & \\
\hline 2.0926 & -465.143230 & 0942 & 244 & 10418 & 4 & 2.09012 & 22 & 838 & & & & & \\
\hline 2.1926 & -465.142412 & 2.1942 & -465.117505 & 2.20418 & -465.114229 & 2.19012 & -465.11546 & 2.1838 & 509 & 2.18216 & & 17 & 551 \\
\hline 2.2926 & -465.141498 & 2.2942 & -465.115826 & 2.30418 & -465 & 2.29012 & -465 & 2.2838 & & 2.28216 & & & \\
\hline 2.3926 & -465.140570 & 2.3942 & -465 & 2. & -465 & 2.35 & -465 & 838 & 13 & 16 & 80 & & 357 \\
\hline 2.4926 & -465.1 & 2.4942 & -465 & 2.50418 & -465 & 2.49012 & & & & & & & \\
\hline 2.5926 & -465.138903 & 2.5942 & -465.111330 & 2.60418 & -465. & 2.5 & -465 & 838 & 55 & 16 & 40 & & 492 \\
\hline 2.6926 & -465.138209 & 2.6942 & -465.110097 & 2.70418 & -465.106 & 2.69012 & -465 & 2.6838 & & 2.68216 & & & -46 \\
\hline 2.7926 & -465.137625 & 2.7942 & -465.109002 & 2.80418 & -465. & 2.79012 & -465 & 2.7838 & -465.12753 & 2.78216 & 3560 & 2.7817 & -465 \\
\hline 2.8926 & -465.1 & 2.8942 & -465. & 2.90418 & -465 & 2.89012 & & 838 & 674 & 2.88216 & & & 2406 \\
\hline 2.9926 & -465.136790 & 2.9942 & -465.107083 & 3.00418 & -465.103329 & 2.99012 & -465.10514 & 2.9838 & -465.12614 & 2.98216 & -465.121930 & 2.9817 & -465.121691 \\
\hline 3.0926 & -465.136552 & 3.0942 & -465.106146 & 3.10418 & -465.103277 & 3.09012 & -465.10417 & 3.0838 & -465.12562 & 3.08216 & -465.121220 & 3.0817 & -465.120963 \\
\hline 3.1926 & -465.136328 & 3.1942 & -465.105223 & 3.20418 & -465.102541 & 3.19012 & -465.10316 & 3.1838 & -465.12512 & 3.18216 & -465.120537 & 3.1817 & -465.120244 \\
\hline 3.2926 & -465.136099 & 3.2942 & -465.104329 & 3.30418 & -465.101860 & 3.29012 & -465.10216 & 3.2838 & -465.12464 & 3.28216 & -465.119860 & 3.2817 & -465.119554 \\
\hline 3.3926 & -465. & 42 & & 3.4 & & 3.3 & -465 & 38 & 9 & 3.3 & -465.119211 & 3.3817 & -465.118901 \\
\hline 3.4926 & -465.136032 & 3.4942 & -465.102688 & 3.50418 & -465.100671 & 3.49012 & -465.10021 & 3.4838 & -465.12378 & 3.48216 & -465.118593 & 3.4817 & -465.118264 \\
\hline
\end{tabular}


Figure S1. The relative energies from the relaxed PE scans of Table S17.

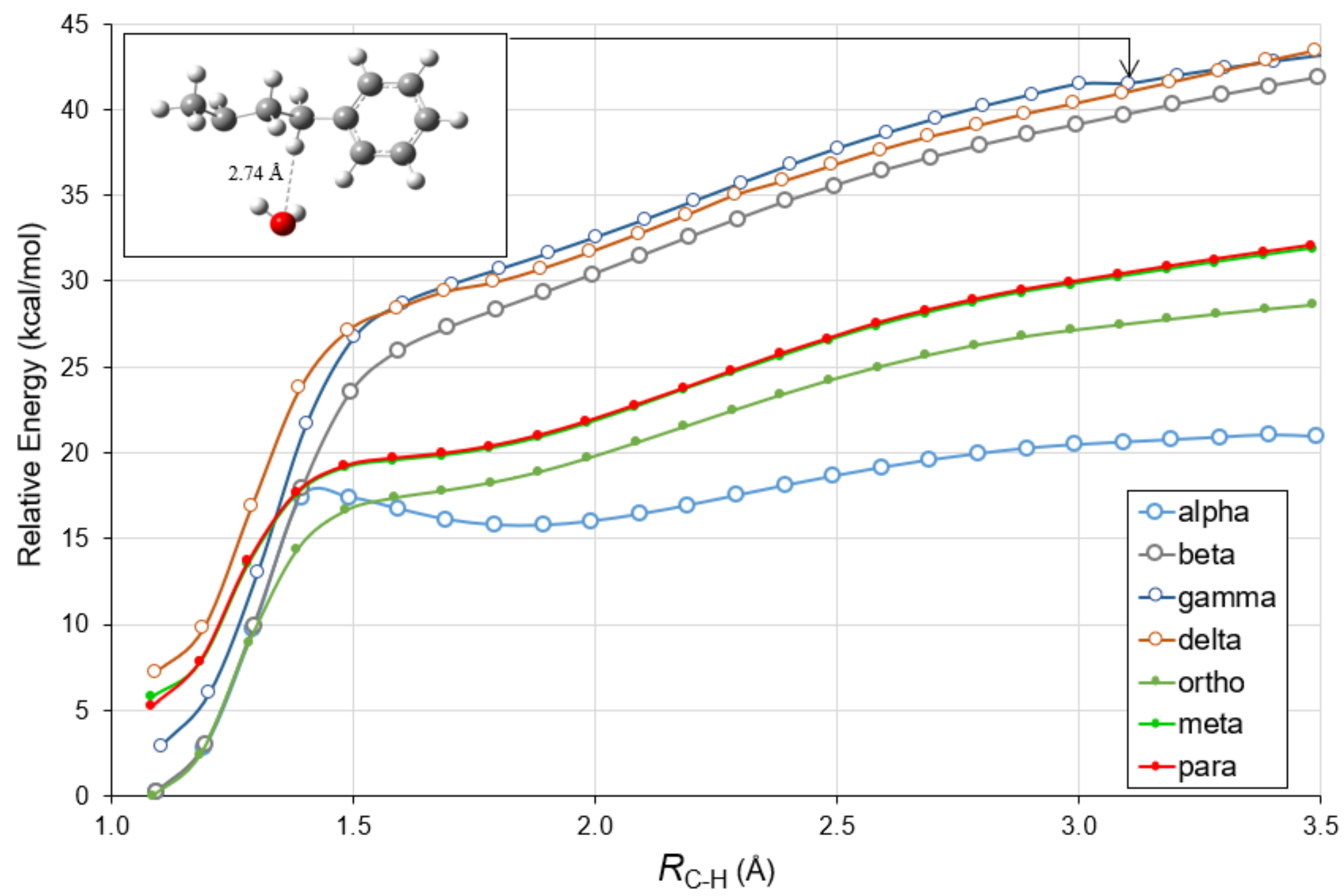

The small kink in the case of the $\gamma$-proton PE curve at 3.1 A is caused by the $\mathrm{H}_{2} \mathrm{O}$ molecule formed from the $\gamma$-proton coordinating with the more acidic proton at the $\alpha$-position. 
Figure S2. Additional stabilization of the ortho anion

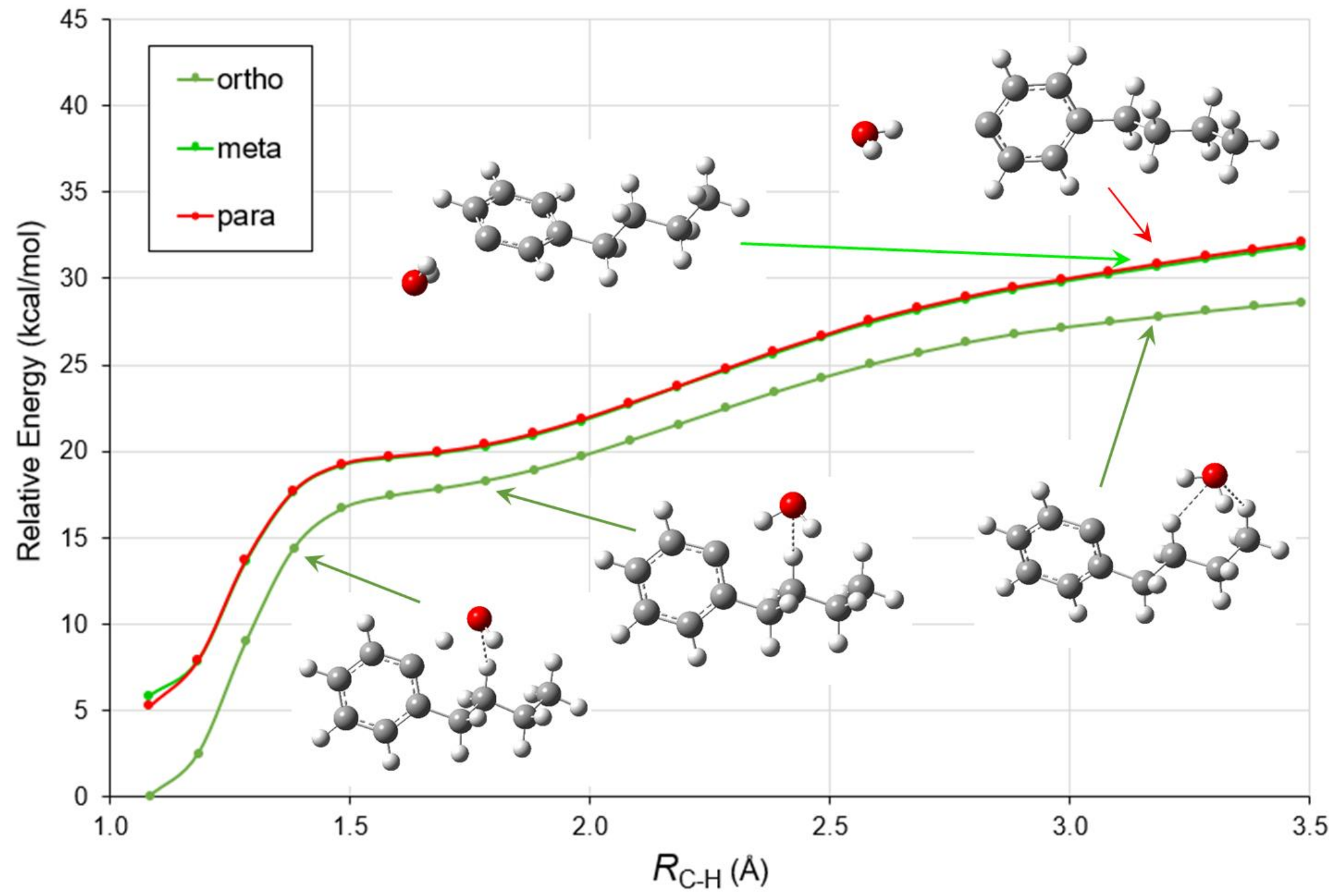

The water formed from the ortho anion hydrogen bonds with various side chain protons as it moves away, providing additional stabilization. This is not available to the meta or para anions, which may explain why their energies are asymptotically higher in Fig. S1 than that of the ortho. 